\title{
Jet Formation and Evolution in Baroclinic Turbulence with Simple Topography
}

\author{
ANDREW F. THOMPSON \\ Department of Applied Mathematics and Theoretical Physics, University of Cambridge, Cambridge, United Kingdom
}

(Manuscript received 15 January 2009, in final form 21 August 2009)

\begin{abstract}
Satellite altimetry and high-resolution ocean models indicate that the Southern Ocean comprises an intricate web of narrow, meandering jets that undergo spontaneous formation, merger, and splitting events, as well as rapid latitude shifts over periods of weeks to months. The role of topography in controlling jet variability is explored using over 100 simulations from a doubly periodic, forced-dissipative, two-layer quasigeostrophic model. The system is forced by a baroclinically unstable, vertically sheared mean flow in a domain that is large enough to accommodate multiple jets. The dependence of (i) meridional jet spacing, (ii) jet variability, and (iii) domain-averaged meridional transport on changes in the length scale and steepness of simple sinusoidal topographical features is analyzed.

The Rhines scale, $\ell_{\beta}=2 \pi \sqrt{V_{e} / \beta}$, where $V_{e}$ is an eddy velocity scale and $\beta$ is the barotropic potential vorticity gradient, measures the meridional extent of eddy mixing by a single jet. The ratio $\ell_{\beta} / \ell_{T}$, where $\ell_{T}$ is the topographic length scale, governs jet behavior. Multiple, steady jets with fixed meridional spacing are observed when $\ell_{\beta} \gg \ell_{T}$ or when $\ell_{\beta} \approx \ell_{T}$. When $\ell_{\beta}<\ell_{T}$, a pattern of perpetual jet formation and jet merger dominates the time evolution of the system. Zonal ridges systematically reduce the domain-averaged meridional transport, while two-dimensional, sinusoidal bumps can increase transport by an order of magnitude or more. For certain parameters, bumpy topography gives rise to periodic oscillations in the jet structure between purely zonal and topographically steered states. In these cases, transport is dominated by bursts of mixing associated with the transition between the two regimes. Topography modifies local potential vorticity (PV) gradients and mean flows; this can generate asymmetric Reynolds stresses about the jet core and can feed back on the conversion of potential energy to kinetic energy through baroclinic instability. Both processes contribute to unsteady jet behavior. It is likely that these processes play a role in the dynamic nature of Southern Ocean jets.
\end{abstract}

\section{Introduction}

Ocean flows are replete with coherent structures on scales ranging from the Rossby deformation radius $\lambda$, tens of kilometers, to the size of ocean basins, many thousands of kilometers. Ocean jets, defined as zonally elongated flows typically exhibiting banded structure with alternating eastward and westward velocities, are an example of coherent structures observed throughout this range of scales. The near-universal presence of jets in the ocean has been substantiated by observations of surface currents from satellite altimetry (Hughes and Ash 2001; Maximenko et al. 2005), as well as from finely resolved

Corresponding author address: Andrew F. Thompson, Dept. of Applied Mathematics and Theoretical Physics, Centre for Mathematical Sciences, Wilberforce Road, Cambridge CB3 0WA, United Kingdom.

E-mail: a.f.thompson@damtp.cam.ac.uk numerical simulations (Nakano and Hasumi 2005). Jets with a remarkably zonal orientation, spanning roughly $3^{\circ}$ of latitude, are found to fill the Pacific and Atlantic Ocean basins outside of equatorial regions (Richards et al. 2006; Kamenkovich et al. 2009). However, these structures only appear after sufficiently long time averaging.

In marked contrast, smaller-scale jets appear explicitly in instantaneous images of Southern Ocean velocity fields obtained from both observations (Sokolov and Rintoul 2007) and eddy-resolving numerical models [e.g., the Ocean Circulation Climate Advanced Modelling (OCCAM) $1 / 12^{\circ}$ model, Lee and Coward (2003); the Modeling Eddies in the Southern Oscillation (MESO) project, Hallberg and Gnanadesikan (2006)]. These jets are distinct from ocean basin jets in that they are thin, ribbonlike features that undergo significant meandering. The locations of these jets are largely fixed by topographical features; however, over periods of weeks to months, jets may form and disappear, merge and split, 
and shift latitudes rapidly (Sokolov and Rintoul 2007; S. Thorpe and D. Stevens 2009, unpublished manuscript). The existence of these jets relies on the unique properties of the Antarctic Circumpolar Current (ACC). The absence of continental boundaries across the latitudes spanning the Drake Passage allows a stratification and circulation to develop that (a) has a larger zonal mean flow and (b) is more sensitive to mesoscale eddies and topographical features than ocean basins [see reviews by Rintoul et al. (2001) and Olbers et al. (2004)]. This produces an environment where zonal jets play a similar role to atmospheric storm tracks (Williams et al. 2007). Still, the dynamics that set the jets' horizontal length scales, time scales of variability, and velocity amplitudes are still being investigated. Not surprisingly, then, an understanding of how these jets influence the largescale transport of heat and tracers across the ACC is also underdeveloped.

While the ACC's circumpolar flow is the primary mechanism for exchanging heat, chemicals, dissolved gases, and other tracers between ocean basins, meridional transport across the ACC is also of vital importance for determining carbon dioxide distributions (Le Quéré et al. 2007), the global heat balance (Hogg et al. 2008; Gille 2008), and the export of dense water formed around the margins of Antarctica (Orsi et al. 2001). Thus, ocean circulation or coupled ocean-atmosphere climate models must accurately incorporate meridional transport processes in the ACC. This requires a representation (i.e., resolution or parameterization) of mesoscale eddies and jets, which can be as small as the deformation radius $\lambda$, roughly $10-20 \mathrm{~km}$ in the ACC (Chelton et al. 1998).

A complicating factor in modeling the ACC is the lack of consensus about the dynamically relevant scales with regard to transport. At one extreme, Marshall et al. (2006) calculate eddy diffusivities from tracer fields advected by observed surface velocities and conclude that the ACC acts as a single large-scale circumpolar transport barrier. This result differs from the traditional view that the ACC is composed of three strong fronts that can be traced continuously around Antarctica following specific water mass properties (Orsi et al. 1995; Belkin and Gordon 1996). Recently, satellite altimetry has provided a more intricate view of the ACC's structure, with analysis of gradients in sea surface height or sea surface temperature indicating that the ACC is dominated by a complex web of filamentary structures (Hughes and Ash 2001; Dong et al. 2006; Sokolov and Rintoul 2007). Sokolov and Rintoul (2007) have shown that rather than 3 circumpolar jets, $6-10$ individual narrow jets may be detected across the ACC at a given time and longitude.
Eastward jets in the ACC are typically associated with barriers to meridional transport. ${ }^{1}$ Thus, the distinction between circumpolar jets and more intricate webbed or braided structures may be crucial for large-scale transport estimates. Figure 1 shows snapshots of layer-wise potential vorticity (PV) from two doubly periodic quasigeostrophic (QG) turbulence simulations on a $\beta$ plane (i.e., with a large-scale PV gradient): one without and one with topography (top and bottom panels, respectively). In the former case, regions of nearly homogeneous PV develop between strong eastward jets, which focus along sharp meridional gradients in PV (Rhines 1994; Dritschel and McIntyre 2008). In the case with topography, a set of well-mixed PV regions is still apparent, although the boundaries separating these regions undergo large meridional excursions due to topographic steering. If these boundaries, or barriers, remain coherent, then topographic steering of the jets has little effect on the large-scale meridional transport, and mixing across the entire domain remains limited. If, however, jet steering results in other processes, such as eddy shedding or a local breakdown of transport barriers, this may alter the large-scale transport. Note that lower-layer PV gradients are sharper in the simulation with topography due to local enhancements of the background PV gradients.

This study focuses on the role that simple topographies play in setting the jet structure in a large domain with many jets. Specifically, a suite of over 100 simulations of equilibrated baroclinic turbulence in a two-layer, doubly periodic QG model are used to address (a) the meridional scaling of the jets, (b) the spatial and temporal variabilities of the jet structure, and (c) the resulting domain-averaged meridional transport characteristics. The simulations are performed in a domain that is large compared to $\lambda$ so that, like the ACC, many jets may form. Following the results of Pavan and Held (1996), a doubly periodic domain provides an accurate assessment of the dynamics far from the boundaries. QG models of the ACC with topography have been explored by Treguier and Hua (1988), Treguier and McWilliams (1990), and Wolff et al. (1991), among others. In these studies, the emphasis was on quantifying the energetics of the system, especially the role of bottom form stress. The studies of Vallis and Maltrud (1993), Treguier and Panetta (1994), Sinha and Richards (1999), and Hogg and Blundell (2006) consider how topography influences jet structure, although either due to the type of model used or because of the parameter regime explored, they were unable to capture the range of patterns of behavior described

\footnotetext{
${ }^{1}$ It has recently been shown that a jet's resilience to mixing may have complicated vertical variability (Greenslade and Haynes 2008; Smith and Marshall 2009). In these studies jets are more effective barriers to transport in upper layers.
} 
(a)

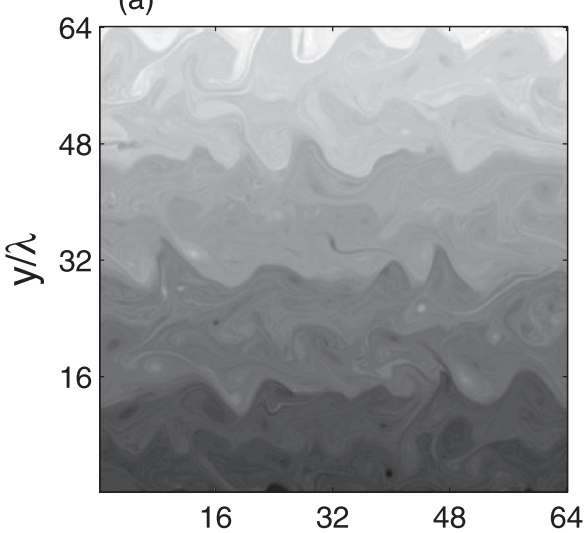

(c)

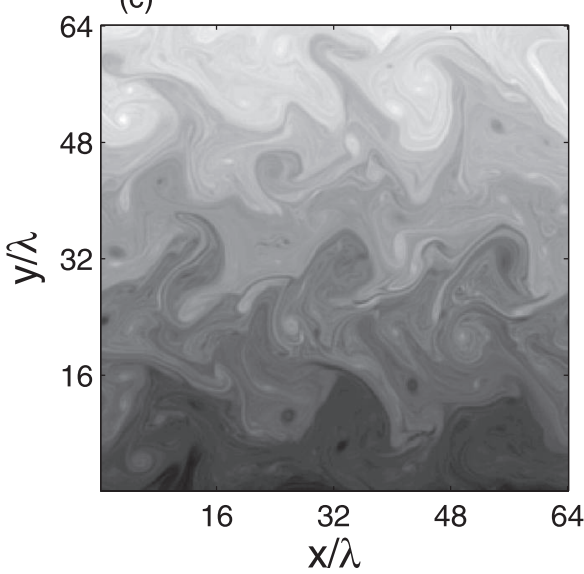

(b)

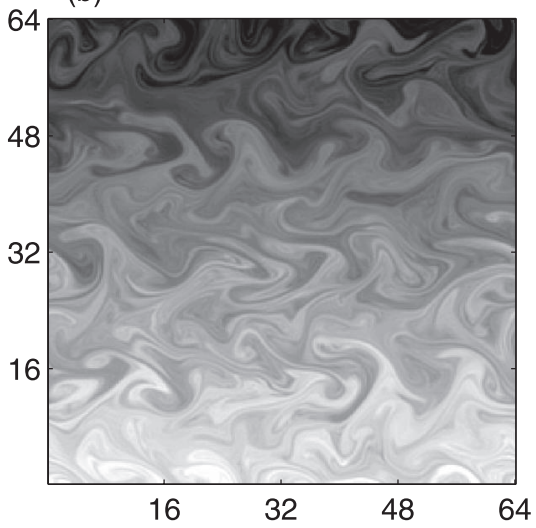

(d)

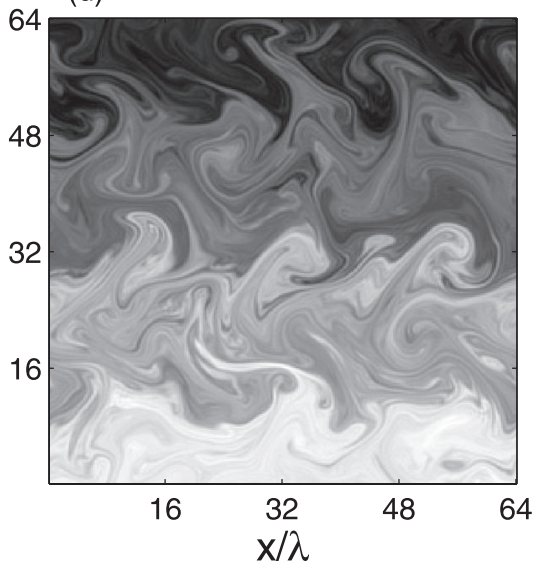

FIG. 1. Snapshots of PV in the (a) upper and (b) lower layers of a quasigeostrophic simulation with a flat bottom; the parameters are $\beta=0.75$ and $\kappa=0.1$ (see section 2a for details). (c),(d) Snapshots of PV in the upper and lower layers, respectively, of a simulation with bumpy bottom topography. The topography is defined by a series of sinusoidal bumps as in (19) with $k_{T}=6 \pi / 64 \lambda$ and $s=1.0$ (Fig. 2). The topography causes the lower-layer meridional PV gradient to vary between $-1.25 U / \lambda^{2}$ and $0.75 U / \lambda^{2}$. All other parameters are the same as in the top panels.

here. Furthermore, the current study considers the implications of jet variability on large-scale transport properties.

Section 2 briefly describes the model and two diagnostics used to analyze the flow: the Rhines scale, $\ell_{\beta}$, and the effective diffusivity, $\kappa_{\text {eff }}$. Section 3 considers the jet structure in simulations with three types of topography: bottom slopes, zonally invariant ridges, and twodimensional arrays of sinusoidal bumps. A discussion of the mechanisms contributing to the jet variability, as well as a consideration of the applicability of this idealized model to Southern Ocean flows, appears in section 4. Conclusions are given in section 5.

\section{The model and diagnostics}

\section{a. Equations}

We consider the two-layer, rigid-lid quasigeostrophic model on a $\beta$ plane in a doubly periodic domain with simple topography (Pedlosky 1987; Panetta 1993). The choice of a two-layer model is based on the ease of numerical calculation, allowing a systematic exploration of parameter space, while also allowing the system to be forced "physically" through baroclinic instability. Potential vorticity is advected by a $2 \mathrm{D}$ incompressible flow in each layer and experiences dissipation at small and large scales:

$$
\frac{\partial}{\partial t} Q_{i}+J\left(\Psi_{i}, Q_{i}\right)=-\kappa^{*} \delta_{i 2} \nabla^{2} \Psi_{2}+\text { ssd }
$$

Here, $\Psi_{i}$ is the streamfunction of the upper $(i=1)$ and lower $(i=2)$ layers, $J$ is the horizontal Jacobian, $\delta_{i j}$ is the Kronecker delta, $\nabla^{2}$ is the horizontal Laplacian operator, and ssd is the small scale dissipation needed for numerical stability. Small-scale dissipation is implemented using a wavenumber filter as described in the appendix of Smith et al. (2002). The potential vorticities, 
$Q_{i}$, and velocities $\left(u_{i}, v_{i}\right)$ are defined in terms of the streamfunction:

$$
\begin{aligned}
Q_{i} & =\nabla^{2} \Psi_{i}+\frac{(-1)^{i}}{2 \lambda^{2}}\left(\Psi_{1}-\Psi_{2}\right)+\beta^{*} y+\frac{f_{0}}{H} \delta_{i 2} \eta, \\
\left(u_{i}, v_{i}\right) & =\left(-\frac{\partial \Psi}{\partial y}, \frac{\partial \Psi}{\partial x}\right) .
\end{aligned}
$$

Here, $\lambda$ is the (internal) deformation radius, the Coriolis frequency is approximated by $f=f_{0}+\beta^{*} y, H$ is the mean layer depth (taken to be the same for the top and bottom layers), and the total depth of the lower layer is $H-\eta$. The Ekman damping coefficient is given by $\kappa^{*}=$ $f_{0} d_{E} / 2 H$, where $d_{E}$ is the Ekman-layer depth.

The total streamfunctions are given by $\Psi_{1}=-2 U y+$ $\psi_{1}^{*}$ and $\Psi_{2}=\psi_{2}^{*}$. Thus, the source of energy in the model comes from the extraction of potential energy of a stationary, vertically sheared flow through baroclinic instability (see discussion in section $4 \mathrm{~d}$ ). The equations are nondimensionalized using $\lambda$ and $U,\left(\psi_{i}^{*}=U \lambda \psi_{i}, q_{i}^{*}=\right.$ $\left.U q_{i} / \lambda\right)$, which yields, dropping the ssd term,

$$
\begin{aligned}
q_{1 t}+\psi_{1 x}(\beta+1)+2 q_{1 x}+J\left(\psi_{1}, q_{1}\right) & =0, \\
q_{2 t}+\psi_{2 x}(\beta-1)+J\left(\psi_{2}, q_{2}+h\right) & =-\kappa \nabla^{2} \psi_{2},
\end{aligned}
$$

where $\kappa=\kappa^{*} \lambda / U$ and $\beta=\beta^{*} \lambda^{2} / U$ are nondimensional parameters $q_{i}+\nabla^{2} \phi_{i}+(-1)\left(\phi_{1}-\phi_{2}\right) / 2$ are the perturbation potential vorticities and $h(x, y)=f_{0} \lambda \eta / U H$. In the case with a uniform bottom slope

$$
h=\frac{s y}{\lambda}, \quad s=\alpha \frac{f_{0} \lambda^{2}}{U H}, \quad \alpha=\text { const. }
$$

and the term $(\beta-1)$ in (5) becomes $(\beta-1+s)$.

The modal forms of the equations are obtained using the relationships

$$
\psi=\frac{\psi_{1}+\psi_{2}}{2}, \quad \tau=\frac{\psi_{1}-\psi_{2}}{2},
$$

where $\psi$ and $\tau$ are the perturbation streamfunctions of the barotropic and baroclinic modes, respectively. The energy balance is obtained by multiplying $\psi_{1}$ to (4) and $\psi_{2}$ to (5) and integrating over both turbulent fluctuations and the doubly periodic domain, indicated by \langle\rangle , which in a statistical steady state gives

$$
\left\langle\psi_{1} \psi_{2 x}\right\rangle=\kappa\left\langle\left|\nabla \psi_{2}\right|^{2}\right\rangle+\text { ssd. }
$$

The contribution of the ssd term to the right-hand side of (8) is never more than a few percent in these simulations. Using (7),

$$
\left\langle\psi_{1} \psi_{2 x}\right\rangle=2\left\langle\psi_{x} \tau\right\rangle
$$

such that the domain-averaged heat, or thickness, flux $\left\langle\psi_{x} \tau\right\rangle$ is proportional to the energy production (cf. Larichev and Held 1995; Thompson and Young 2006).

Examples of the topographies considered in this study are given in Fig. 2. Figure 2d gives an example of how topographical features locally modify the barotropic PV and its gradient. For all simulations discussed in section 3 , $\kappa=0.1$ and the domain size is square with length $L=64 \lambda$ and grid size is $256^{2}$.

\section{b. Diagnostics}

\section{1) EFFECTIVE DIFFUSIVITY}

One diagnostic employed to analyze jet structure and transport in these simulations is the effective diffusivity $\kappa_{\text {eff }}$, introduced by Nakamura (1996). Although primarily used in atmospheric contexts to indicate mixing regions and barriers to isentropic eddy transport (e.g., Haynes and Shuckburgh 2000; Allen and Nakamura 2001), more recently, Marshall et al. (2006) have used effective diffusivities to quantify eddy diffusivities in the Southern Ocean within a stream-wise framework. A careful derivation of the effective diffusivity calculation is given in Shuckburgh and Haynes (2003) and Nakamura (2008a).

The magnitude of $\kappa_{\text {eff }}$ is a measure of tracer contour complexity with the implicit assumption that stirring by eddies is responsible for increasing tracer complexity. Transport barriers are associated with simple tracer contours and therefore small values of $\kappa_{\text {eff }}$, while regions of large $\kappa_{\text {eff }}$ represent strong mixing. The values of $\kappa_{\text {eff }}$ shown in the present study are not meant to imply any quantitative similarity to eddy processes in the ocean or atmosphere. Instead, the emphasis is on quantifying how different topographies induce relative variations in transport properties. Thus, the nondimensional effective diffusivity values shown here simply reflect the ratio of the square of the stirred contour length $L_{\mathrm{eq}}$ to the square of the minimum contour length, or equivalently the domain width $L$ :

$$
\kappa_{\mathrm{eff}}\left(y_{e}, t\right)=\frac{L_{\mathrm{eq}}^{2}\left(y_{e}, t\right)}{L^{2}} .
$$

Here, $y_{e}$ is an equivalent latitude, which is the latitude a given contour would have if it were remapped to be zonally symmetric, while retaining its internal area (Shuckburgh and Haynes 2003).

Throughout this study the effective diffusivity calculations are made using contours of upper-layer PV $Q_{1}$. From (1), it can be seen that, neglecting ssd, $Q_{1}$ is 

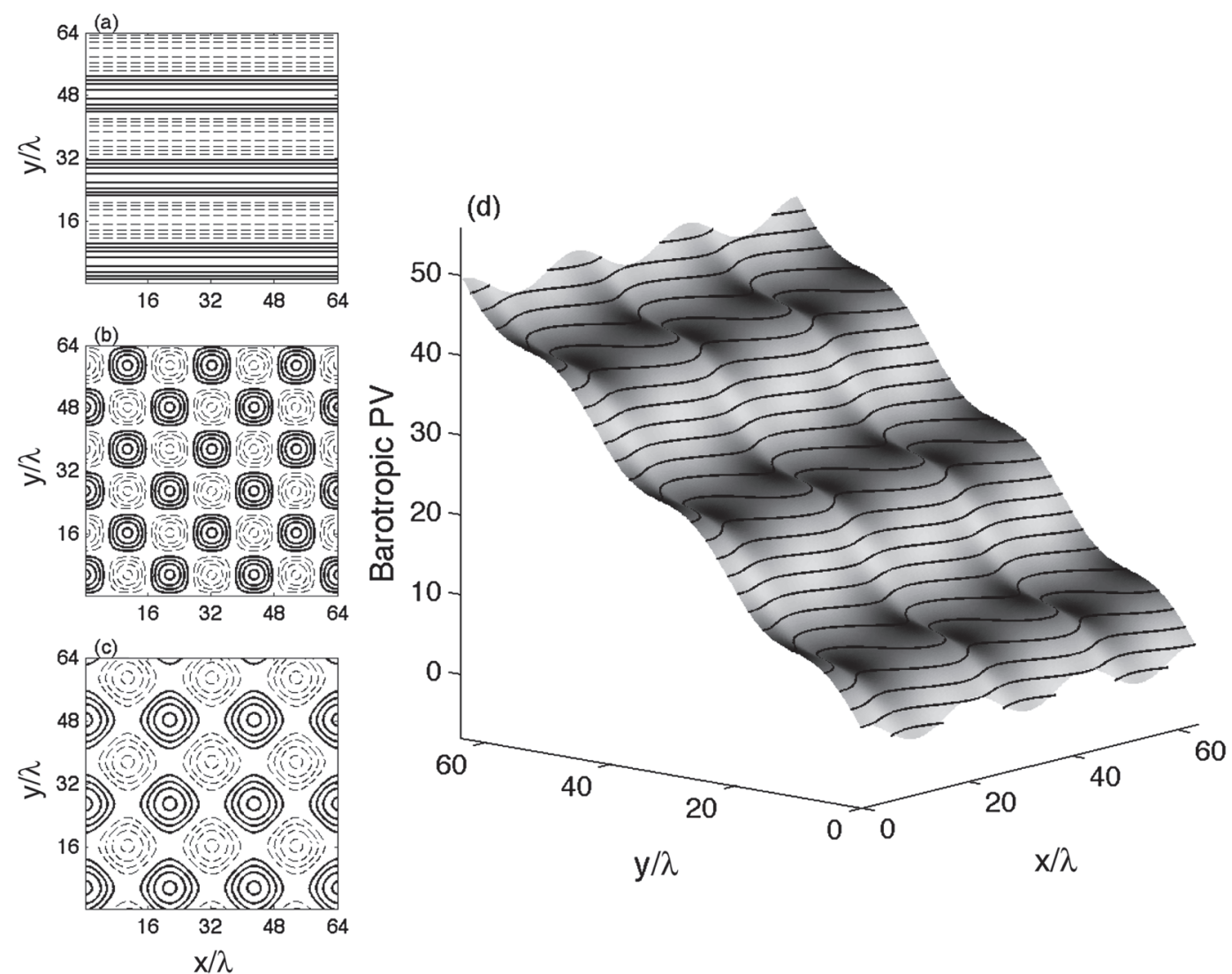

FIG. 2. Examples of bottom topographies considered in this study. They include (a) zonally invariant sinusoidal ridges (14), (b) aligned sinusoidal bumps with zero zonal/meridional means (18), and (c) unaligned sinusoidal bumps with nonzero zonal-meridional means (19). Solid and dashed contours indicate rises and depressions, respectively. (d) An indication of how topography modifies the nondimensional barotropic $\mathrm{PV}, Q_{\mathrm{BT}} \lambda / U$, given by the surface and contours, for a simulation with $\beta=0.75, s=1$.0. Shading indicates the magnitude of $\left|\nabla\left(Q_{\mathrm{BT}} \lambda / U\right)\right|$. This is the same topography as is used in Figs. $1 \mathrm{c}$ and $1 \mathrm{~d}$; here, the meridional, barotropic PV gradient varies between 0.25 and $1.25 U / \lambda^{2}$.

advected by the total upper-layer streamfunction $\Psi_{1}$ and is materially conserved. Thus, contours of $Q_{1}$ produce the same distribution of $\kappa_{\text {eff }}$ as would a passive tracer maintained by a background gradient. In other words, modification of the streamfunction through PV inversion at each time step influences a passive tracer and $Q_{1}$ in the same manner. Lower-layer PV $Q_{2}$, on the other hand, is also modified by bottom friction, and for this reason only upper-layer $\kappa_{\text {eff }}$ distributions are considered. It is shown in section 3 that regions of low $\kappa_{\text {eff }}$ in the upper layer accurately track the position of the barotropic jets (see also Haynes et al. 2007). The distribution of $\kappa_{\text {eff }}$ for a reference flat-bottom case with multiple jets is discussed in section $3 b$.

\section{2) RHINES SCALING}

The Rhines scale, $\ell_{\beta}$, is the crucial characteristic of the flow that determines its interaction with topography. This section reviews the dependence of $\ell_{\beta}$ in simulations without topography to changes in the parameters $\beta$ and $\kappa$. The data are taken from the simulations described in Thompson and Young (2007), who revisited the work of Panetta (1993) with the ability to significantly increase the domain size and horizontal resolution. Since the domain is doubly periodic, the simulations must select an integer number of jets; thus, extending the domain size is an important check on a number of the features described in Panetta (1993). Thompson and Young (2007) use a slightly different form of bottom friction than described in section $2 a$, which does not significantly influence the behavior of the system.

The jet spacing, $\ell_{J}$, is estimated by counting the number of jets $\left(n_{J}\right)$ in the physical fields of the simulations. In most cases $n_{J}$ is unambiguous, although cases arise where $n_{J}$ is not clearly defined as it transitions from one integer to another. In this case half values are permitted (e.g., $n_{J}=5.5$ ). Figures $3 \mathrm{a}$ and $3 \mathrm{~b}$ provide a complete survey of the equilibrated value of $n_{J}$ and show that 

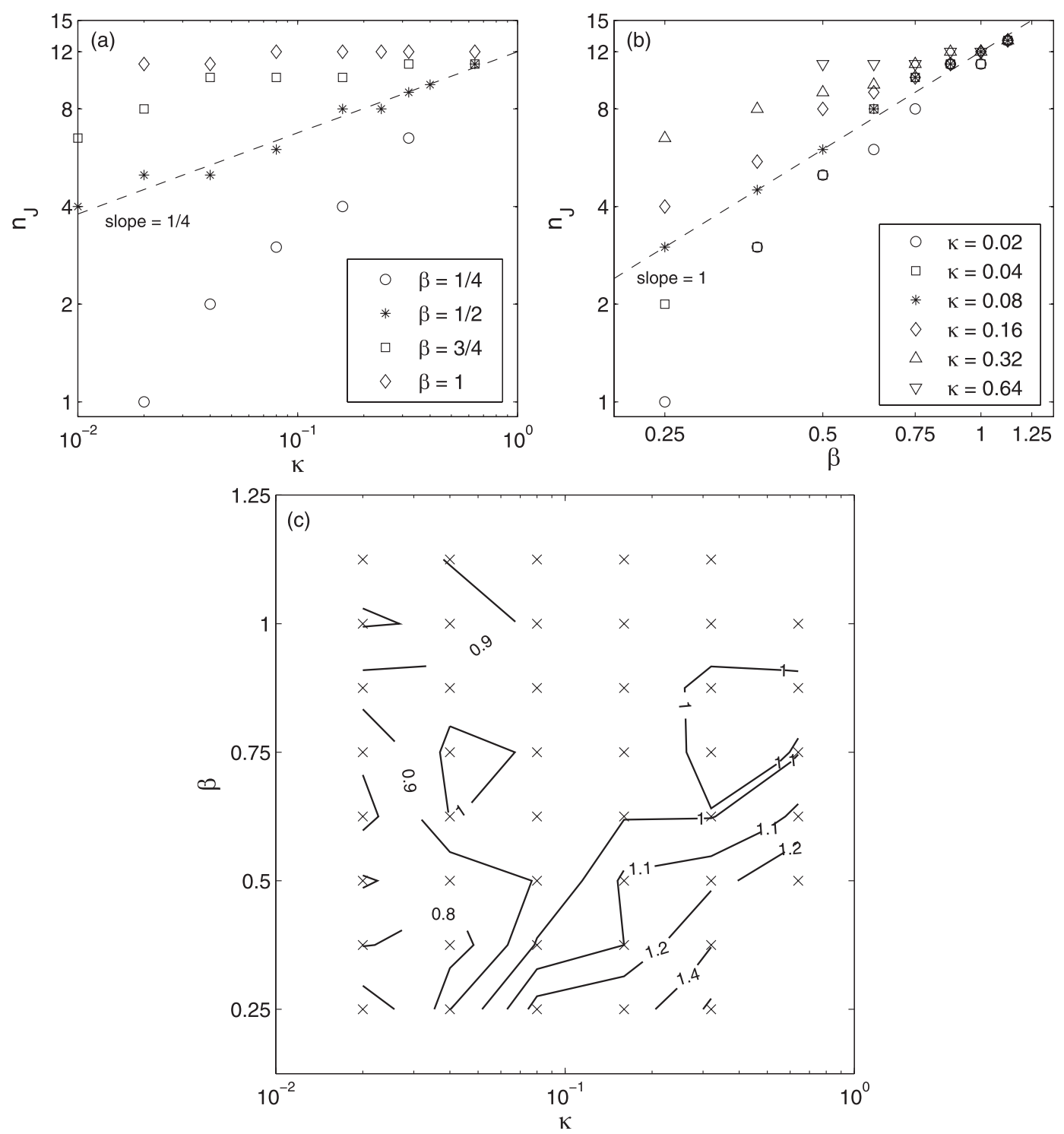

FIG. 3. Survey of the equilibrated number of jets $n_{J}$ in a square domain with sides of length $2 \pi(25 \lambda)$ as a function of nondimensional (a) bottom friction $\kappa=\kappa^{*} \lambda / U$ and (b) PV gradient $\beta=\beta^{*} \lambda^{2} / U$ for simulations with a flat bottom [data from Thompson and Young (2007)]. (c) Contours of the ratio $\ell_{\beta} / \ell_{J}$ for the same simulations. The Rhines scale $\ell_{\beta}$ is defined in (11) and (12), and $\ell_{J}=L / n_{J}$, where $L$ is domain size and $n_{J}$ is determined from the observed number of jets. The crosses mark parameters where data are available.

the dependence on $\kappa$ and $\beta$ is not a simple power law. For example, for the case $\beta=1 / 2, n_{J}$ increases roughly like $\kappa^{1 / 4}$ (Fig. 3a), while for $\kappa=0.08, n_{J}$ grows linearly with $\beta$ (Fig. 3b). However, Figs. 3a and $3 \mathrm{~b}$ taken together indicate that these simple scaling laws are special and do not apply except along these particular slices through parameter space; that is, $n_{J}$ does not scale like $\beta \kappa^{1 / 4}$.

Although $n_{J}$ is too complicated to be described by a simple power law involving the external parameters $\beta$ and $\kappa$, the hope is that $n_{J}$ can simply be related to other equilibrated statistics of the flow. Rhines scaling sug- gests that the jet spacing should scale like $(\text { velocity } / \beta)^{1 / 2}$ Panetta (1993) suggested that an eddy velocity should be applied such that

$$
\ell_{\beta}=2 \pi \sqrt{V_{e} / \beta},
$$

where

$$
V_{e}=\sqrt{\left\langle\left|\nabla \psi_{1}^{\prime}\right|^{2}+\left|\nabla \psi_{2}^{\prime}\right|^{2}\right\rangle}
$$


Here and throughout, the primes indicate that the zonal mean has been removed; that is, $\psi_{i}^{\prime}=\psi_{i}-\overline{\psi_{i}}$. At small values of bottom friction, Panetta (1993) found good correlation between $\ell_{\beta}$ and $\ell_{J}$ (see his Fig. 4). As further confirmation, Fig. $3 \mathrm{c}$ shows contours of the ratio $\ell_{\beta} / \ell_{J}$ over a range of $\kappa$ and $\beta$ values. The ratio $\ell_{\beta} / \ell_{J}$ drifts away from 1 as the jets become weaker, specifically in simulations with small $\beta$ and large $\kappa$. In simulations where both $\beta$ and $\kappa$ are weak, as few as two jets occupy the domain, and quantization is likely responsible for the poorer agreement between $\ell_{\beta}$ and $\ell_{J}$ in this corner of parameter space. Rhines scaling was confirmed in a more realistic ocean model by Sinha and Richards (1999) using a similar definition of $\ell_{\beta}$. Alternatively, Berloff et al. (2009) find that their quasigeostrophic simulations do not support Rhines scaling. Possible reasons for this discrepancy include the following: the dominance of lateral eddy viscosity in the Berloff model as compared to bottom friction, variable layer depths in the Berloff model as compared to equal layer depths here, and the presence of meridional boundaries in the Berloff model. All of these differences and their subsequent modifications to Rhines scaling arguments would be worthy of a more dedicated study.

The main conclusion of this section is that Panetta's version of Rhines's scaling is successful at condensing the results over a wide range of $\beta$ and $\kappa$ values, which is remarkable given the complex dependence of $n_{J}$ on $\kappa$ and $\beta$ summarized in Fig. 3. Importantly, the agreement between $\ell_{\beta}$ and $\ell_{J}$ implies that $\ell_{\beta}$ represents the meridional extent of well-mixed PV regions in equilibrated baroclinic turbulence with a large-scale PV gradient (cf. Fig. 1).

\section{Results}

\section{a. Bottom slope}

In layer-wise QG models, topography modifies the PV gradient in a single, lower layer (although all modal PV gradients are affected). Another key layer-wise process is the forcing of the zonal mean flow by Reynolds stress correlations. This process, which generates upgradient momentum fluxes (Held and Andrews 1983) and nonlocal spectral transfers (Huang and Robinson 1998), was shown by Thompson and Young (2007) to occur almost exclusively in the upper layer of a two-layer model. Since the modification of PV gradients by topography and the maintenance of the jets by Reynolds stresses occur in different layers, it is not immediately clear how topography might affect meridional jet scales. Thus, the first consideration is how the jet structure and meridio- nal scaling respond to the varying steepness of a uniform, meridional bottom slope $s(6){ }^{2}$

The role of topography in setting the meridional jet scale is examined by considering whether the Rhines scaling $\ell_{\beta}$ continues to predict the jet spacing. In this case $\beta$ must be modified to account for the bottom slope. The best agreement was found by taking the modified $\beta$ to represent the barotropic PV gradient:

$$
\beta_{\mathrm{BT}}=\beta+\frac{s}{2},
$$

which is similar to the result found by Sinha and Richards (1999). Figure 4a shows a survey of the ratio $\ell_{\beta} / \ell_{J}$, where $\ell_{\beta}=2 \pi \sqrt{V_{e} / \beta_{\mathrm{BT}}}$ and $\ell_{J}=L / n_{J}$. Each dot represents a simulation and its size gives the corresponding value of $\ell_{\beta} / \ell_{J}$. With the exception of the simulation with $\beta=0$ and $s=1.5, \ell_{\beta} / \ell_{J}=1 \pm 0.15$, which is comparable to the agreement in the flat-bottom simulations (Fig. 3c). Figures $4 \mathrm{~b}$ and $4 \mathrm{c}$ show how $\ell_{\beta} / \ell_{J}$ and $n_{J}$ depend on $\beta_{\mathrm{BT}}$. Figure $4 \mathrm{c}$ indicates that jet spacing generally decreases with increasing $\beta_{\mathrm{BT}}$; however, for a given $\beta_{\mathrm{BT}}$, different combinations of $\beta$ and $s$ can produce various equilibrated energy levels and jet spacings.

The success of Rhines scaling with $\beta_{\mathrm{BT}}$ indicates that although Reynolds stress forcing of the mean flow is a layer-wise process (Thompson and Young 2007), the barotropic PV gradient ultimately determines the jet scale. This raises the question of how topographies that locally modify PV gradients, but do not alter the domainaveraged PV gradient, influence the equilibrated jet structure.

\section{b. Zonal ridges}

The next level of topographic complexity considered is zonally invariant ( $\partial h / \partial x=0$ everywhere), or zonal, ridges. Zonal ridges mark a departure from most studies of turbulence over topography, which tend to include meridional ridges (e.g., Borowski et al. 2002). This is because meridionally oriented topography can support a topographical form stress, which is a key mechanism for removing momentum from channel-like flows, such as the ACC (Munk and Palmén 1951). The focus on this intermediate case between topographic slopes and twodimensional topographies is to first understand how

\footnotetext{
${ }^{2}$ The condition for linear instability in the case with $\kappa=0$ is that $(\beta+1)$ and $(\beta-1+s)$ have opposite signs (Charney 1971), although with the introduction of bottom friction, the system becomes unstable at values of $\beta$ and $s$ beyond the frictionless critical point (Holopainen 1961). The linear stability problem with a linear slope in the lower layer is studied by Hart (1975) and Steinsaltz (1987).
} 

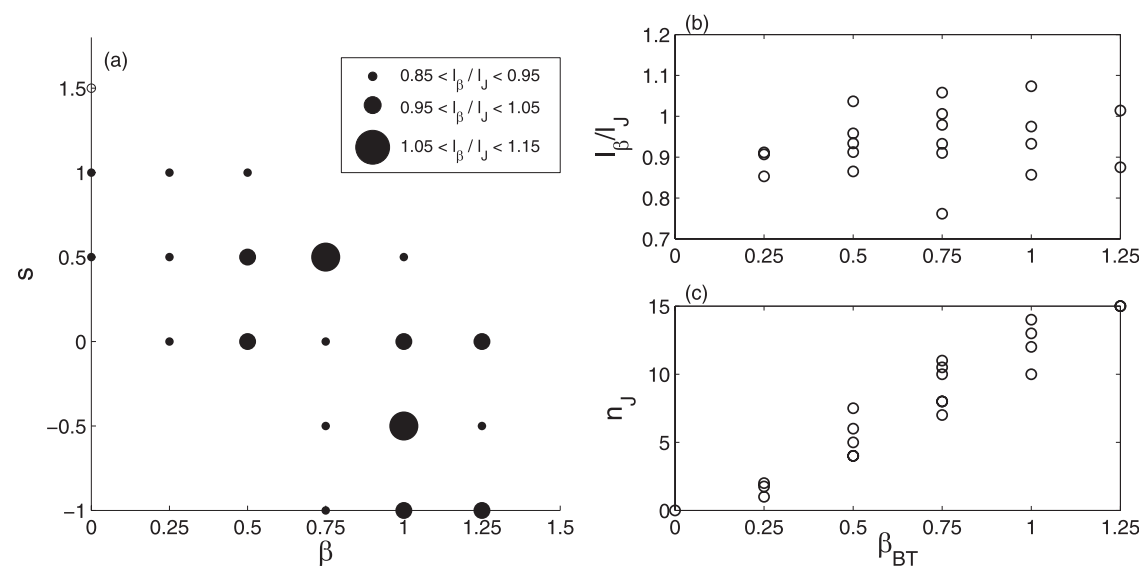

FIG. 4. Survey of the jet scale in simulations with a uniform slope $s$ (6) in the lower layer. (a) The ratio $\ell_{\beta} / \ell_{J}$ (given by the size of the circles) as a function of a nondimensional $\beta$ and the bottom slope $s$. The value of $\ell_{\beta} / \ell_{J}$ for the open circle $(\beta=0, s=1.5)$ is 0.76 ; all other values are within $1 \pm 0.15$. (b) The ratio $\ell_{\beta} / \ell_{J}$ as a function of the barotropic $\mathrm{PV}$ gradient $\beta_{\mathrm{BT}}=\beta+s / 2$. (c) Number of observed jets $n_{J}$ in a $128 \lambda \times 128 \lambda$ domain as a function of $\beta_{\mathrm{BT}}$.

local PV modifications alter equilibrated jet structures, while restricting the mean flow to be zonal.

All zonal ridge simulations were completed with base parameters $\beta=0.75$ and $\kappa=0.1$. In the absence of topography, these parameters produce a statistically equilibrated state with $\ell_{\beta}=16 \lambda$. Therefore, in a domain of size $64 \lambda \times 64 \lambda$, four jets form, which are resolved accurately (see section $4 c$ ) with a computational grid that still permits sampling of a large region of parameter space. Holding $\beta$ and $\kappa$ fixed, two parameters are varied: the number of ridges $n_{R}=L / \ell_{T}$, where $\ell_{T}$ is the wavelength of a single ridge, and a measure of the topographical steepness, $s$. The ridges (nondimensional) are described by

$$
h(y)=\frac{s}{k_{T} \lambda} \sin \left(k_{T} y\right),
$$

where $k_{T}=2 \pi / \ell_{T}$. For $\beta=0.75$, the background PV gradient of the lower layer changes sign in the domain if $s>0.25$, while the barotropic PV gradient only changes sign for $s>1.5$.

Figure 5 summarizes the behavior of the jets by showing a series of Hovmöller (time-latitude) plots of upper-layer $\kappa_{\text {eff }}$ (left panels) and zonally averaged zonal barotropic velocities (right panels) for simulations with varying $n_{R}$ and $s$. Figure 5a shows the structure of $\kappa_{\text {eff }}$ for the $n_{R}=0, s=0$ flat-bottom reference case. Four steady, eastward zonal jets form with weak westward flows occurring between these jets. The structure of $\kappa_{\text {eff }}$ in the upper layer is a series of eight mixing regions and eight transport barriers. Strong mixing regions are found on the flanks of the eastward jets. Cores of the eastward jets are strong barriers to transport, while cores of the westward jets are weaker barriers to transport; $\kappa_{\text {eff }}$ is roughly 4 times greater in westward jet cores than in eastward jet cores. ${ }^{3}$

Figure 5b shows a simulation with $n_{R}=3, s=1.0$ and gives a typical example of the unsteady jet behavior that can develop. Multiple, coherent jets form preferentially in regions where topography enhances $\beta$-where the PV gradient is strongest. The jets then drift toward regions of weaker PV gradient where they dissipate, allowing new jets to form. This results in continuous jet formation and merger, giving rise to a braided jet structure reminiscent of the temporal variability of Southern Ocean jets (cf. Sokolov and Rintoul 2007, Fig. 1). The ratio $\ell_{\beta} / \ell_{T}$ is given above each panel in Fig. 5, where $\ell_{\beta}$ is defined by $2 \pi \sqrt{V_{e} / \beta}$, that is, local PV modifications are neglected. For $\ell_{\beta} / \ell_{T} \approx 1$, the system locks into a steady jet structure (Fig. 5c); however, the equilibrated jet scale $\left(n_{J}=5\right)$ need not be the same as in the flat-bottom case $\left(n_{J}=4\right)$. A pattern of six steady jets can also be obtained with $\ell_{\beta} / \ell_{T}=1.31$. The positions of the jets in Fig. $5 \mathrm{c}$ are fixed by the ridges with jets located where the topographic slope is steepest. Eastward jets are now found where topography opposes $\beta$. As the scale of the topography is reduced, $\ell_{\beta} / \ell_{T}>1$ and the system reverts to a steady pattern with $n_{J}=4$ (Fig. 5d). Figure 5e shows that the unsteady braided structure is a robust feature, even for relatively shallow topographies. Figure $5 \mathrm{f}$ shows that wide and steep topographies can support mixed

\footnotetext{
${ }^{3}$ Transport barriers coinciding with the westward jets give support to recent work by Beron-Vera et al. (2008), who have found similar barriers in westward jets in the atmosphere.
} 

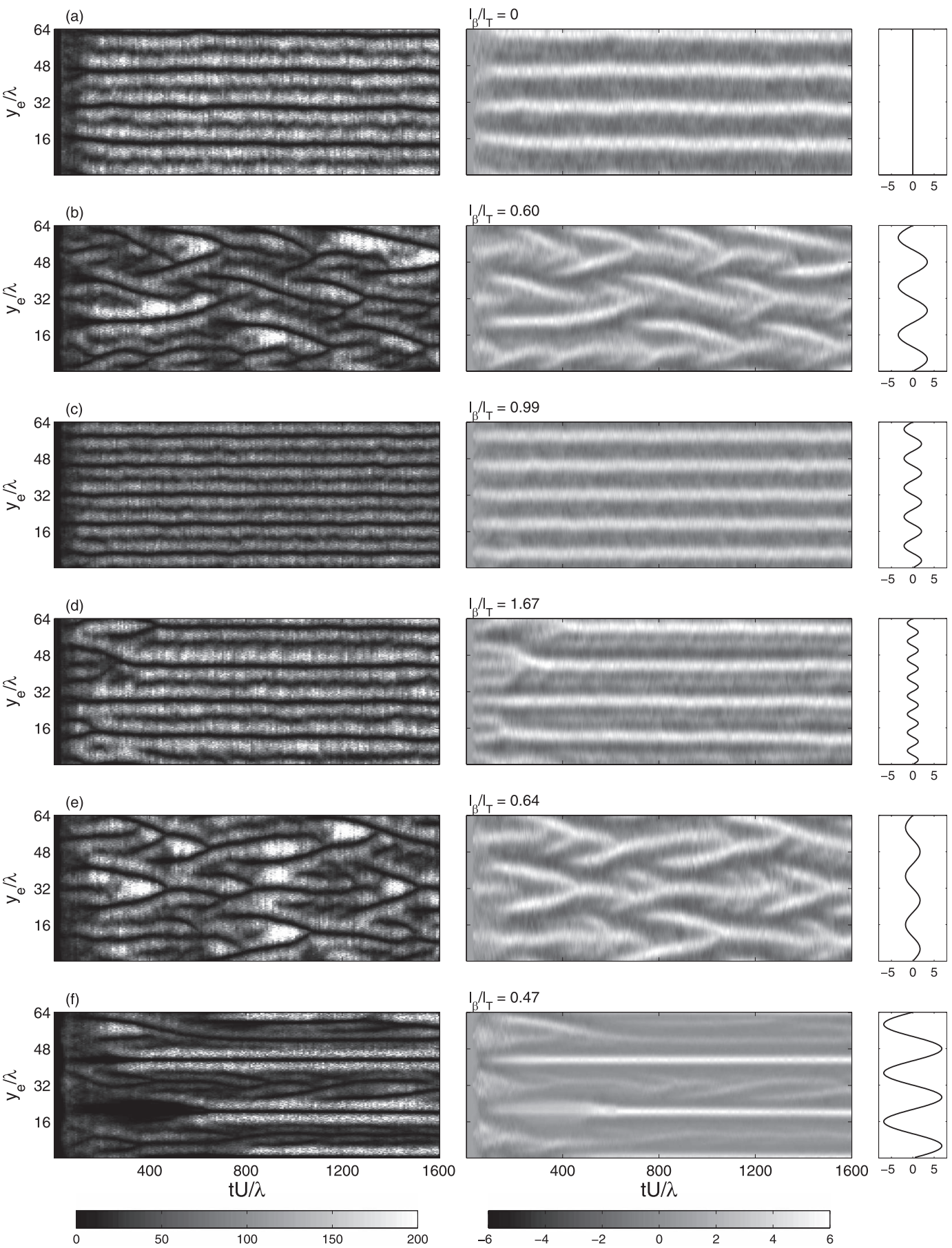

FIG. 5. Survey of zonal ridge simulations: (left) time series of effective diffusivity $\kappa_{\text {eff }}$ calculated from the upperlayer potential vorticity field, (middle) time series of the zonally averaged, zonal barotropic velocity, and (right) the meridional profile of the topography in the lower layer. In the left panels the ordinate is an equivalent latitude [see (10)], while for the other panels the ordinate is true latitude $y / \lambda$. The parameter values are (a) $n_{R}=0, s=0$; (b) $n_{R}=$ $3, s=1.0$; (c) $n_{R}=5, s=1.0 ;$ (d) $n_{R}=8, s=1.0 ;$ (e) $n_{R}=3, s=0.5$; and (f) $n_{R}=3, s=2.0$. The ratio $\ell_{\beta} / \ell_{T}$ is given for each set of panels. 

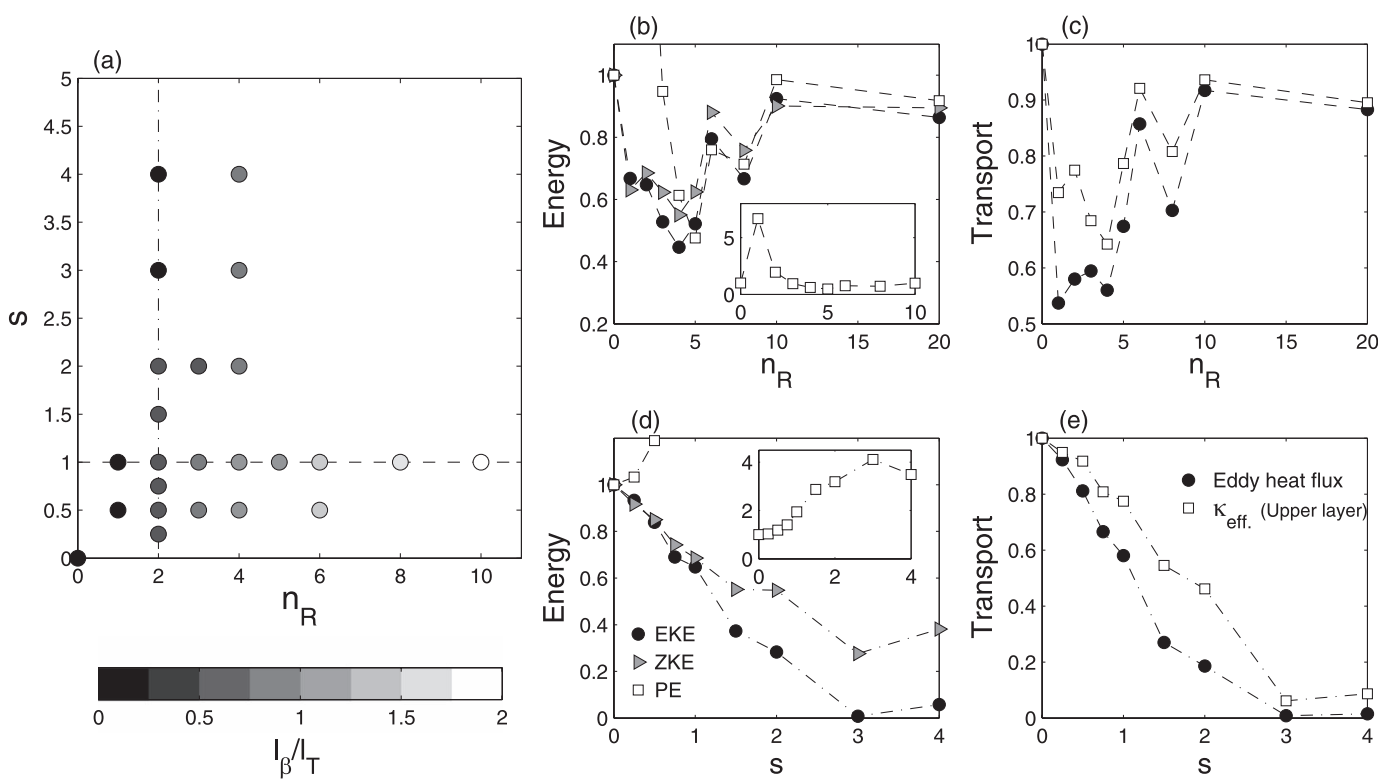

FIG. 6. Survey of the statistics from the simulations with zonal ridges. (a) The ratio $\ell_{\beta} / \ell_{T}$ (given by the shade) as a function of ridge number, $n_{R}=L / \ell_{T}$, and ridge steepness, $s$ (14). (b),(c) The $s=1.0$ data are shown along the horizontal dashed line; (d),(e) the $n_{R}=2$ data are shown along the vertical dashed-dotted line. In all cases, $\beta=0.75$, $\kappa=0.1$, and $L / \lambda=64$. (b),(d) Time and domain-averaged values of EKE (○), ZKE ( $\triangleright)$, and PE ( $\square$ ), defined in (15). The inset plots in (b) and (d) show PE values and have the same axes labels as their corresponding panels. (c),(e) Time and domain-averaged values of HF $(\circ)$ and $\varkappa_{\text {eff }}(\square)$, defined in (16). Domain-averaged statistics in (b)-(e) have been normalized by the statistical properties of a simulation with a flat bottom $\left(n_{R}=0\right.$ or $\left.s=0\right)$.

behavior with weak unsteady braided jets (e.g., along $\left.y_{e} / \lambda=32\right)$ occurring between strong steady jets.

Examining $\ell_{\beta} / \ell_{T}$ further, when $\ell_{\beta} / \ell_{T}>1$, turbulent mixing occurs over horizontal scales greater than the scales of local PV modifications. The eddies essentially smear out the PV signature of the topography and the structure of the jets is observed to be similar to the flatbottom case. For $\ell_{\beta} / \ell_{T} \approx 1$, the jets are steady and fixed by the scale of the topography. For $\ell_{\beta} / \ell_{T}<1$, the system feels the local PV modifications imposed by the ridges. For $0.5<\ell_{\beta} / \ell_{T}<1$, a braided structure is observed throughout the domain, while for $\ell_{\beta} / \ell_{T}<0.5$, a combination of steady and braided jets is typically observed. Note that variations in $s$ alter energy levels and modify $\ell_{\beta}$; thus, the ratio $\ell_{\beta} / \ell_{T}$ is the key parameter determining the behavior of the jets.

Figure 6 describes how changes in jet structure, responding to variations in $n_{R}$ and $s$, modify the domainaveraged energetics and meridional transport of the system. Figure $6 \mathrm{a}$ gives the ratio $\ell_{\beta} / \ell_{T}$ for all the simulations completed in the ridge configuration. Two parameter values, indicated by the dashed lines, are explored in detail: $n_{R}=2$ and $s=1.0$.

Figures $6 \mathrm{~b}$ and $6 \mathrm{~d}$ show time and domain-averaged values of the eddy kinetic energy (EKE), zonal kinetic energy (ZKE), and potential energy (PE), where

$$
\begin{aligned}
\mathrm{EKE} & =\frac{1}{2}\left\langle\left|\nabla \psi_{1}^{\prime}\right|^{2}+\left|\nabla \psi_{2}^{\prime}\right|^{2}\right\rangle, \quad \mathrm{ZKE}=\frac{1}{2}\left\langle\bar{\psi}_{1 y}^{2}+\bar{\psi}_{2 y}^{2}\right\rangle \\
\mathrm{PE} & =\frac{1}{2}\left\langle\left(\frac{\psi_{1}-\psi_{2}}{2}\right)^{2}\right\rangle .
\end{aligned}
$$

The values are normalized by the nondimensional EKE, ZKE, and PE values obtained from a simulation with $n_{R}=s=0,[8.95,5.75,3.76]$. Figure $6 \mathrm{~b}$ shows that for fixed $s=1.0$, the kinetic energy levels are maximized when there are no ridges, and they decrease as $n_{R}$ increases up to 4 . The $n_{R}=4$ simulation marks a minimum in both EKE and ZKE at about half the flat-bottom value; the jets have a braided structure in this simulation. Energy levels rise rapidly for $n_{R}=5$ and 6 and remain largely unchanged for $n_{R}>6$, where $\ell_{\beta} / \ell_{T}>1$. For small $n_{R}$, PE jumps to over 5 times the flat-bottom value, but for $n_{R} \geq 4$, the PE ratio falls to values less than 1 , and for larger values of $n_{R}$ trends in PE follow EKE and ZKE. For $n_{R} \geq 10$, the energy ratios are approximately unity, indicating that domainaveraged statistics are similar to flat-bottom values for small-scale topography.

Similar behavior is observed in estimates of the domainaveraged transport characteristics (Figs. 6c and 6e). Two quantities are calculated to examine this, the eddy 

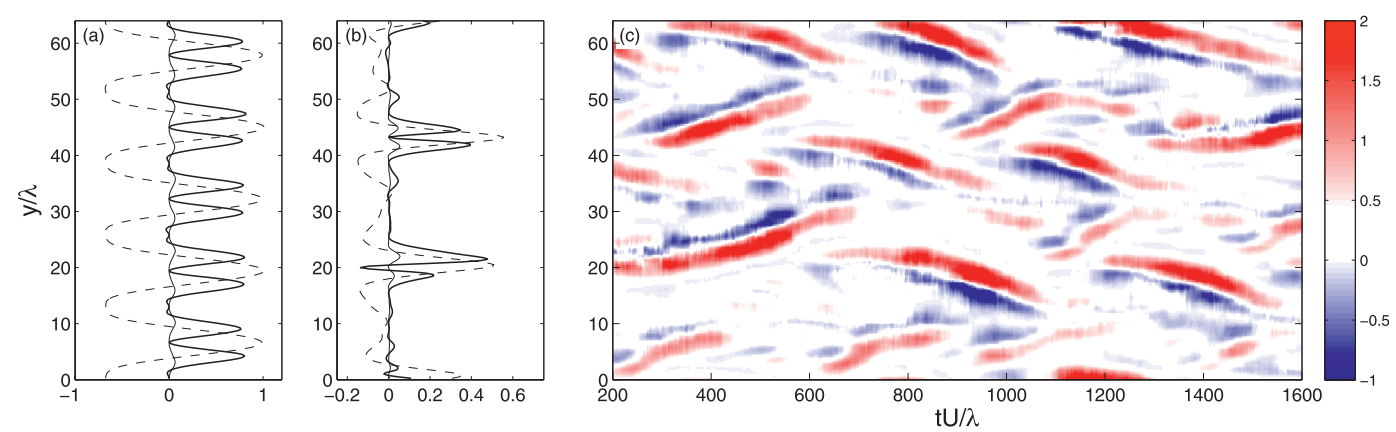

FIG. 7. Time and zonal averages of the Reynolds stress forcing of the zonal mean flow, $\mathcal{R}_{i}(17)$, by upper-layer eddies (boldface curve) and lower-layer eddies (thin curve) in two simulations: (a) $n_{R}=5, s=1.0$ and (b) $n_{R}=3$, $s=2.0$ (Figs. 5c and 5f). The zonally averaged zonal barotropic velocity is given by the dashed lines. (c) Time series of $\mathcal{R}_{1}$ in a simulation with $n_{R}=3, s=1.0$ (Fig. 5b). The values are smoothed with a running mean of duration $50 \lambda / U$.

heat flux (9), HF, and the domain-averaged effective diffusivity $\varkappa_{\text {eff: }}$

$$
\mathrm{HF}=\left\langle\psi_{x} \tau\right\rangle, \quad \varkappa_{\mathrm{eff}}=\left\langle\frac{1}{\overline{\kappa_{\mathrm{eff}}^{-1}} y_{e}}\right\rangle,
$$

where $\varkappa_{\text {eff }}$ is the meridional harmonic mean of $\kappa_{\text {eff }}\left(y_{e}, t\right)$ followed by a temporal mean over the turbulent fluctuations. The harmonic mean is the standard averaging technique for quantifying transport in the presence of transport barriers (cf. Nakamura 2008b). Similar to kinetic energy, topographical features with length scales greater than $\ell_{\beta}$ can reduce domain-averaged transport estimates by nearly a factor of $1 / 2$, but for topographical scales smaller than $\ell_{\beta}$, transport properties revert back to flat-bottom levels. A key point, though, is that both energy and transport ratios have a nonmonotonic dependence on $n_{R}$. The data have three clear local minima at $n_{R}=1,4$, and 8 that are related to changes in the jet structure as the wavelength of the topography is modified.

In contrast, when $n_{R}$ is held fixed $\left(n_{R}=2\right)$ and $s$ increases, EKE and ZKE (Fig. 6d), and HF and $\varkappa_{\text {eff }}$ (Fig. 6e), decrease monotonically. Note that the combination of large $s$ and small $n_{R}$ pushes the limits of QG theory, which requires $\eta$ to remain small compared to the layer depth $H$. Conversely, PE increases smoothly as topography becomes steeper, indicating that the system becomes more stable and less energy is released through baroclinic instability. In simulations with large steepness, the flow is almost purely zonal as ZKE $\gg$ EKE. This monotonic reduction in transport as $s$ increases indicates that the stabilizing flank of a zonal ridge, where $s$ enhances $\beta$, regulates the domain-averaged transport. In other words, if larger, more energetic eddies develop in regions of reduced $\mathrm{PV}$ gradient, they are unable to break down the strong transport barriers that develop in regions of enhanced PV gradient.
This section concludes with a consideration of how mean flow forcing by Reynolds stresses is affected by local modifications to the PV gradients. Figure 7 shows the value $\mathcal{R}$, defined to be the time-averaged correlation between the zonally averaged Reynolds stress and the meridional gradient of the zonal mean flow,

$$
\mathcal{R}_{i}(y) \equiv \bar{u}_{i y}\left(\overline{u_{i} v_{i}}\right)
$$

for three different simulations corresponding to Figs. 5c, 5f, and 5b. Typically, where $\mathcal{R}>0$, there is convergence of eastward momentum in the eastward jets, which is indicative of energy transfer from eddies to the mean flow. In Fig. 7a there are five steady zonal jets imposed by the topography since $\ell_{\beta} / \ell_{T} \approx 1$. As in the flat-bottom case (Thompson and Young 2007), $\mathcal{R}$ dominates in the upper layer (boldface curve) and is weak in the lower layer (thin curve); $\mathcal{R}_{1}$ is large on the flanks of the jet and is symmetric about the jet core (dashed curve). This occurs because the meridional scale of the jet is the same as the topographical scale, such that both flanks of the jet feel the same local PV gradient. Steeper topographies act to localize the $\mathcal{R}$ terms (Fig. $7 b$ ), creating a zonal velocity profile that is significantly different from the flat-bottom scenario. Finally, Fig. 7c shows a Hovmöller plot of $\mathcal{R}_{1}$ in a simulation with a braided jet structure, where the time averaging is now a running mean of duration $50 \lambda / U$. Mixing regions on the flanks of the jets feel different local PV gradients, resulting in asymmetric forcing of the mean flow. The jets are accelerated on one flank and decelerated on the other, which steers the jet across the mean PV gradient. This steering encourages jet merger and produces wide homogenized regions where new jets may form.

\section{c. Bumps}

Topography is now allowed to vary zonally as well as meridionally. Local modifications to PV gradients will 
influence jet structures; however, a new feature is the generation of nonzonal mean flows due to topographic steering. Steering is a result of PV conservation as fluid parcels act to minimize vortex stretching that occurs over topographical rises and depressions (Holloway 1987; Vallis and Maltrud 1993). These modified mean flows will then feed back on the baroclinic instability process (Arbic and Flierl 2004). The results are based on a suite of simulations that keep the parameters $\beta=0.75, \kappa=0.1$ fixed, while varying the two parameters $n_{B}=L / \ell_{T}$, where $\ell_{T}=2 \pi / k_{T}$ is the wavelength of a bump, and the bump steepness $s$. Two (nondimensional) topographic forms are considered: aligned and unaligned bumps given by

$$
\begin{aligned}
h_{\text {aligned }}(x, y) & =\frac{s}{k_{T} \lambda} \sin \left(k_{T} y\right) \cos \left(k_{T} x\right), \quad \text { and } \\
h_{\text {unaligned }}(x, y) & =\frac{s}{k_{T} \lambda}\left[\sin \left(k_{T} y\right)+\cos \left(k_{T} x\right)\right],
\end{aligned}
$$

(see Fig. 2). The aligned topography has zero zonal and meridional means, while the unaligned topography retains zonally averaged and meridionally averaged structures.

Jet behavior patterns are again summarized in a series of Hovmöller plots of upper-layer $\kappa_{\text {eff }}$ for the unaligned topographies (Fig. 8, left). The behavior of the aligned topography cases is qualitatively similar and a separate plot is not included. The middle panels in Fig. 8 again show Hovmöller plots of zonally averaged zonal barotropic velocities; the correspondence with the left-hand panels is weaker in some cases due to the effects of topographic steering. A number of patterns of behavior observed in the zonal ridge configuration are repeated, including braided jets for $\ell_{\beta} / \ell_{T}<1$ (Fig. 8b) and quasisteady jets with spacing set by the topographic scale (i.e., $\ell_{\beta} \approx \ell_{T}$; Fig. 8c). For the latter case, the jet spacing is greater than in the flat-bottom simulation, and these jets may also have a significant nonzonal component due to topographic steering. A new pattern of behavior is observed in Fig. 8d, where at fixed latitudes, strong but transient transport barriers spontaneously form and then give way to strong mixing in a continuous process. The position of the strongest barriers are observed to "jump" throughout the domain, although they are constrained to one of three latitudes by topography (here, $n_{B}=3$ ). As before, the jets recover their flat-bottom structure for large $n_{B}$ and $s \leq 1.0$ (Fig. 8e). However, for steeper topography with many bumps, the topographical scale appears in the upper-layer jet structure, and the barriers associated with westward jets are lost (Fig. 8f).

Figures 9 and 10 show surveys of the domain-averaged statistics for the unaligned and aligned topographies. The statistics are the same as those defined for Fig. 6, except EKE has been replaced by the total kinetic energy: KE $=1 / 2\left\langle\left|\nabla \psi_{1}\right|^{2}+\left|\nabla \psi_{2}\right|^{2}\right\rangle$. Statistics are again normalized by the flat-bottom values $\left(n_{B}=s=0\right)$. The domain-averaged statistics for the unaligned and aligned topographies have qualitatively the same behavior, although the unaligned topography has slightly larger variability.

For a fixed value of $s$, ratios of KE, ZKE, PE, HF, and $\varkappa_{\text {eff }}$ all have peaks at an intermediate value of $n_{B}$ that depends on the magnitude of $s$. For steep topography $(s=2)$ peak values occur for long topographical features $\left(n_{B}=2\right)$, while for shallower topography $(s=1)$ the peak occurs for shorter topographical features $\left(n_{B}=4\right)$. In general, PE is peaked at smaller values of $n_{B}$ than the kinetic energies. At large $n_{B}$ the statistical properties return to flat-bottom values as in the ridge simulations.

Energy levels and domain-averaged transport show a monotonic increase with increasing $s$ for a fixed value of $n_{B}$. The trend is moderate for small $s$, but increases rapidly as $s$ approaches $2 \beta$ - the value required to locally eliminate the barotropic PV gradient. For $s>2 \beta$, the statistics plateau; the transition between low- and highenergy states is less abrupt in the aligned topography case. Heat flux ratios ${ }^{4}$ are larger than the $\varkappa_{\text {eff }}$ ratios in the high-energy state, which likely reflects the additional contribution to the heat flux from the standing eddies.

Unlike the ridge simulations, the introduction of bumpy topography uniformly increases statistical properties, with the exception of a decrease in ZKE for small $n_{B}$. The inhibitive effect of topography locally enhancing $\beta$ is no longer limiting because fluid can escape these regions (e.g., on the eastward and westward flanks of a bump). The uniform increase in energy and transport depends largely on the fact that baroclinic instability is more efficient at extracting PE from nonzonal mean flows (Spall 2000; Arbic and Flierl 2004; Smith 2007), as discussed in section $4 \mathrm{a}$.

We now focus on jet behavior in two simulations shown in Figs. 8b and 8d. Figure 11a shows the timelatitude evolution of $\kappa_{\text {eff }}$ as in Fig. 8 b. There is a persistent series of spontaneous jet formation and subsequent merger events. There are also distinct periods when the strength of the transport barriers weakens (e.g., $t U / \lambda \sim$ 550,850 , and 1500). These occurrences are also apparent

\footnotetext{
${ }^{4}$ Here, the total heat flux is plotted, not the eddy heat flux. With a purely zonal topography, $\langle v\rangle=\left\langle\psi_{x}\right\rangle=0$; therefore, $\langle v \tau\rangle=\left\langle\boldsymbol{v}^{\prime} \tau^{\prime}\right\rangle$. Correlations between the mean $v$ and $\tau$ fields can develop in the case with topographic steering. Unfortunately, it is not possible to save sufficient fields to accurately determine the mean field in each of the simulations shown here. Agreement between the HF and $\varkappa_{\text {eff }}$, which does not suffer from this problem, suggests that the behavior of the eddy heat flux is qualitatively similar.
} 

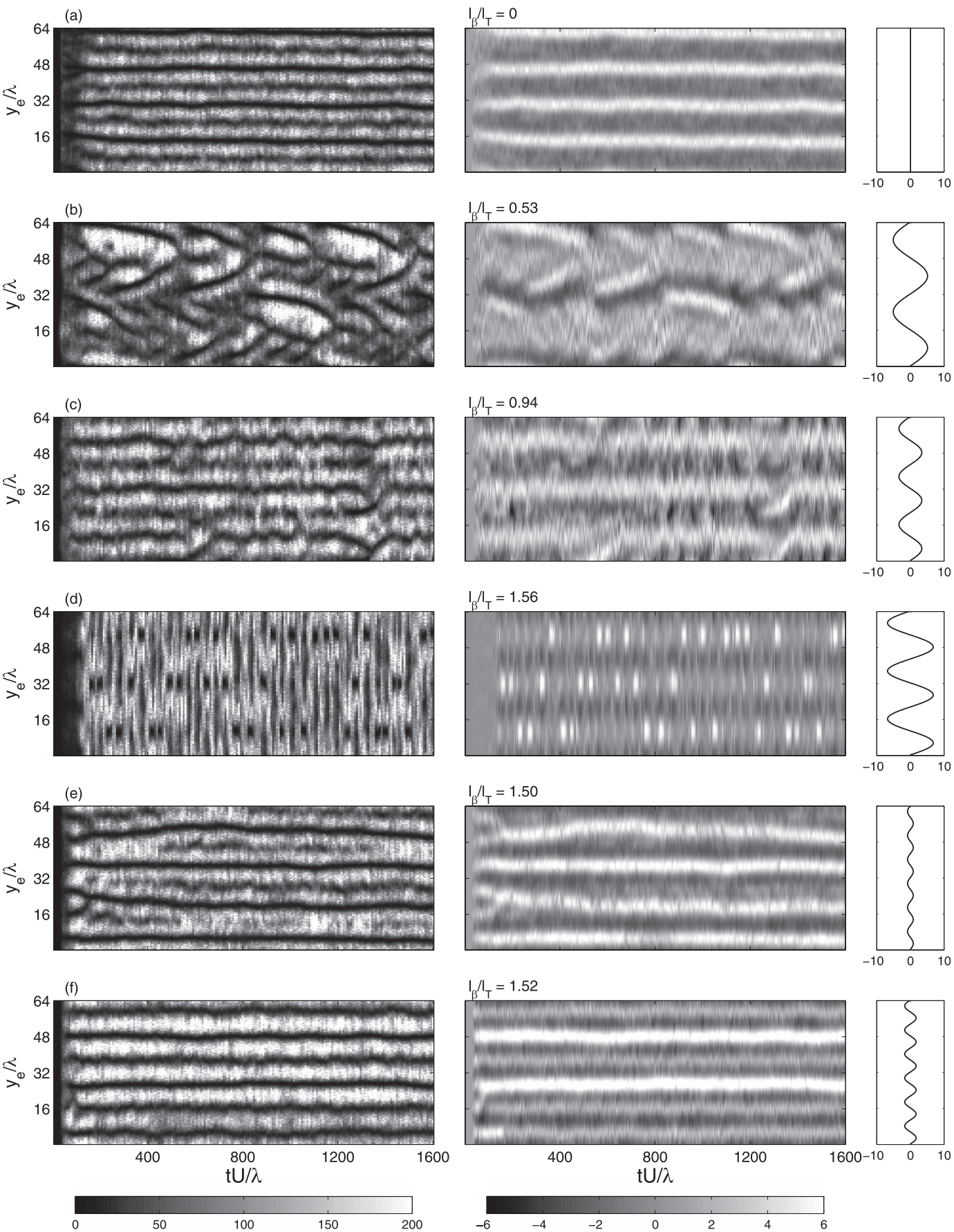

FIG. 8. Survey of simulations with unaligned bumps (19): (left) time series of the effective diffusivity $\kappa_{\text {eff }}$ calculated from the upper-layer potential vorticity field, (middle) time series of the zonally averaged, zonal barotropic velocity, and (right) the meridional profile of the zonally averaged topography in the lower layer. In the left panels the ordinate is an equivalent latitude [see (10)], while for the other panels the ordinate is true latitude $y / \lambda$. The parameter values are (a) $n_{B}=0, s=0$; (b) $n_{B}=2, s=1.0$; (c) $n_{B}=3, s=1.0$; (d) $n_{B}=3, s=2.0$; (e) $n_{B}=6, s=0.5$; and (f) $n_{B}=6, s=$ 1.0. A time-averaged estimate of the ratio $\ell_{\beta} / \ell_{T}$ is given in each set of panels. 

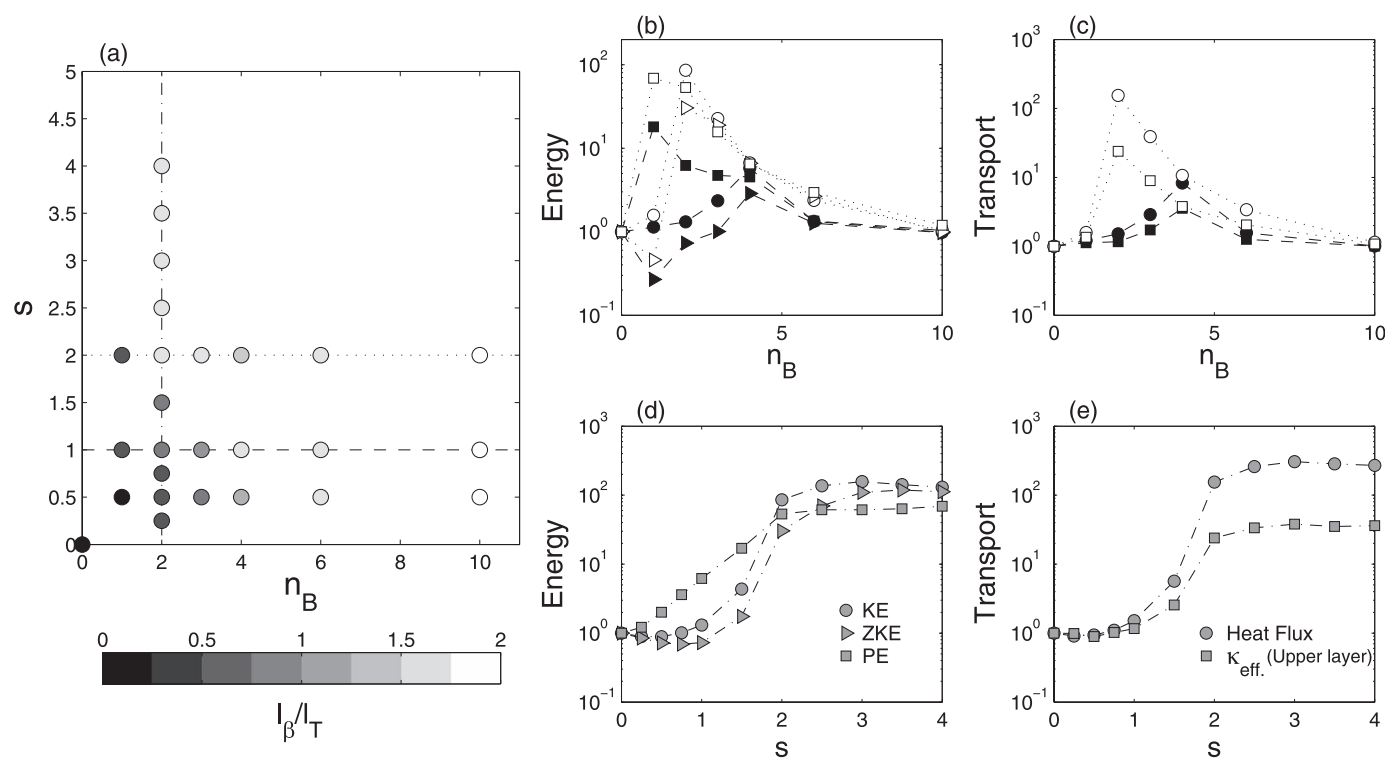

FIG. 9. Survey of the statistics in the simulations with unaligned bumps (19). (a) The ratio $\ell_{\beta} / \ell_{T}$ (given by the shade) as a function of the number of bumps, $n_{B}=L / \ell_{T}$, and bump steepness, $s$ (19). (b),(c) Data for $s=1.0$ are shown along the horizontal dashed line and for $s=2.0$ along the dotted line. (d),(e) Data for $n_{R}=2$ are shown along the vertical dashed-dotted line. In all cases, $\beta=0.75, \kappa=0.1$, and $L / \lambda=64$. (b),(d) Time and domain-averaged values of the KE $(\bigcirc)$, ZKE $(\triangleright)$, and PE $(\square)$. (c),(e) Time and domain-averaged values of the total HF $(\bigcirc)$ and $\varkappa_{\text {eff }}(\square)$, defined in (16). Domain-averaged statistics in (b)-(e) have been normalized by the statistical properties of a simulation with a flat bottom $\left(n_{R}=0\right.$ or $\left.s=0\right)$. Note that the ordinates in (b)-(e) have logarithmic axes, unlike Fig. 6.

in the domain-averaged properties of the system as shown in Fig. 11b, and correspond to peaks in domainaveraged $\mathrm{KE}$ and, to a certain extent, in $\varkappa_{\text {eff }}$ (here the time average has not been performed). Periods of high $\mathrm{KE}$ and $\varkappa_{\text {eff }}$ are typically preceded by a collapse or merger of the transport barriers; the formation of new barriers reduces the energy and mixing rates again. These events occur in a quasiperiodic fashion with a period of roughly $t U / \lambda \geqslant 300$ in this simulation. The formation of new transport barriers can also lead to a shift in the meridional position of enhanced mixing regions (e.g., around $t U / \lambda=850$ ).
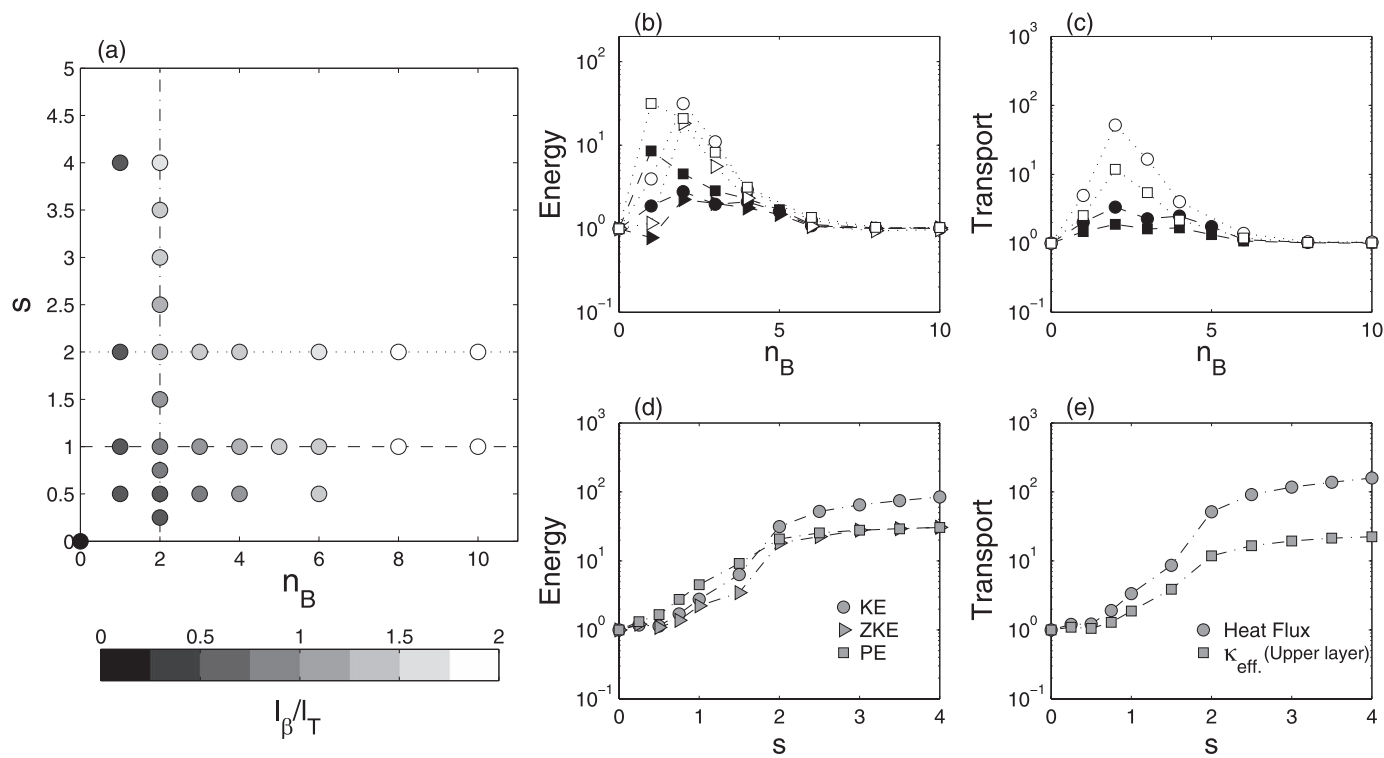

FIG. 10. Same as in Fig. 9 but for simulations with aligned bumpy topography (18). 


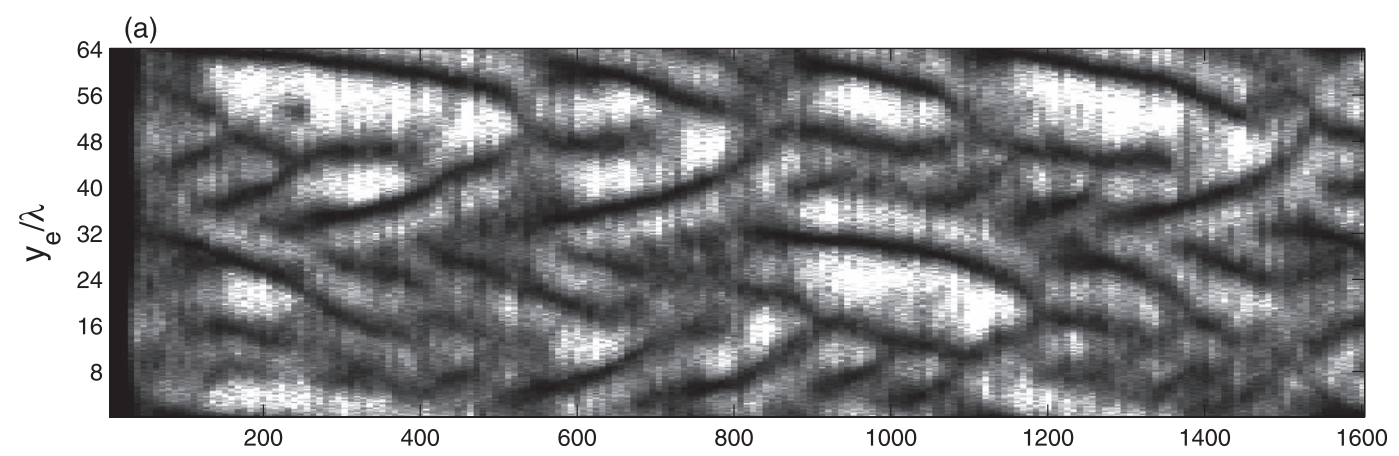

(b)

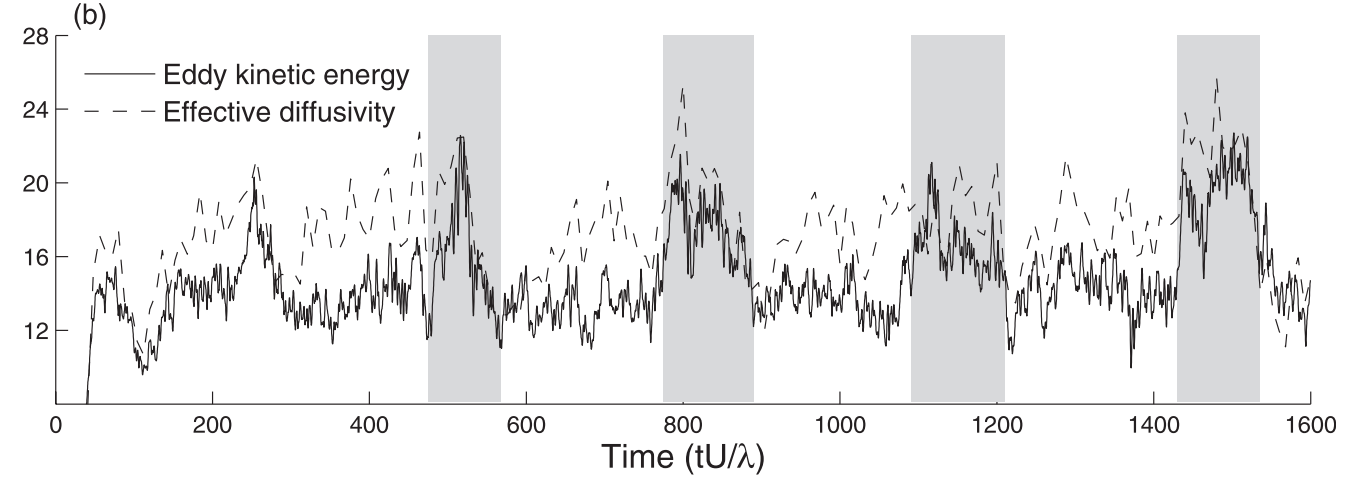

FIG. 11. (a) Time series of the effective diffusivity, $\kappa_{\text {eff }}$, calculated from the upper-layer potential vorticity field in a simulation with unaligned bumps (19) with $n_{B}=2, s=1.0$ (Fig. 8b). (b) The corresponding time series of domainaveraged EKE $=$ KE-ZKE (solid line), and of the meridional harmonic mean of the effective diffusivity, $\varkappa_{\text {eff }}(16)$, (dashed line). The $\varkappa_{\text {eff }}$ time series has been divided by 2 . The gray-shaded areas correspond to significant jet merger events in the top panel.

More dominant periodic behavior may develop in simulations with steeper topography (Fig. 12). Figure 12a shows the time evolution of the domain-averaged KE, ZKE, and PE (corresponding to Fig. 8d). There is a distinct quasiperiodic oscillation in these quantities with a period of approximately $45 \lambda / U$. All three quantities are out of phase, with PE leading, followed by a rapid rise in KE, and finally a peak in ZKE. Similar behavior has been observed in a study by Hogg and Blundell (2006) using a three-layer QG model of the Southern Ocean. Figure $12 \mathrm{~b}$ shows the corresponding zonally averaged zonal barotropic velocity. Although $n_{B}=3$, the Rhines scale $\ell_{\beta}$ is comparable to the domain size during the high-KE state. This results in a single strong jet whose position "jumps" from one topographical feature to another. Each of these shifts is preceded by a period of intense turbulent mixing, which allows the meridional structure to reorganize. There is no discernible trend for how the strong jet selects its latitude (cf. Fig. 8d).

Snapshots of the barotropic vorticity taken at three different times, indicated by the dashed lines, are shown in Figs. 12c-e. During the low-KE-high-PE phase, PV is steered strongly by topography producing a nonzonal mean flow. At the peak KE stage, the eddy scale has grown dramatically and mixing is more vigorous. These large eddies effectively damp the signature of the topography and the flow becomes predominantly zonal. As the KE decays, the eddies become smaller, topography begins to deflect the zonal flow again, and the process repeats itself. This mechanism and its implications for transport in more realistic ocean flows are discussed in section 4 .

\section{Discussion}

\section{a. Variability mechanisms}

In simulations with zonal ridges, unsteady jet behavior involving persistent jet formation and merger, either throughout the domain or in regions between strong steady jets, occurs over a broad region of parameter space. The meridional extent over which PV becomes homogenized in $\beta$-plane, baroclinic turbulence is given by $\ell_{\beta}$ (Dritschel and McIntyre 2008). Thus, if two regions have roughly the same value of $V_{e}$ (12), but topography opposes $\beta$ in one region and enhances $\beta$ in the other, $\ell_{\beta}$ will be larger in the first region (11). In other words, one flank of a ridge generates larger eddies that mix over 

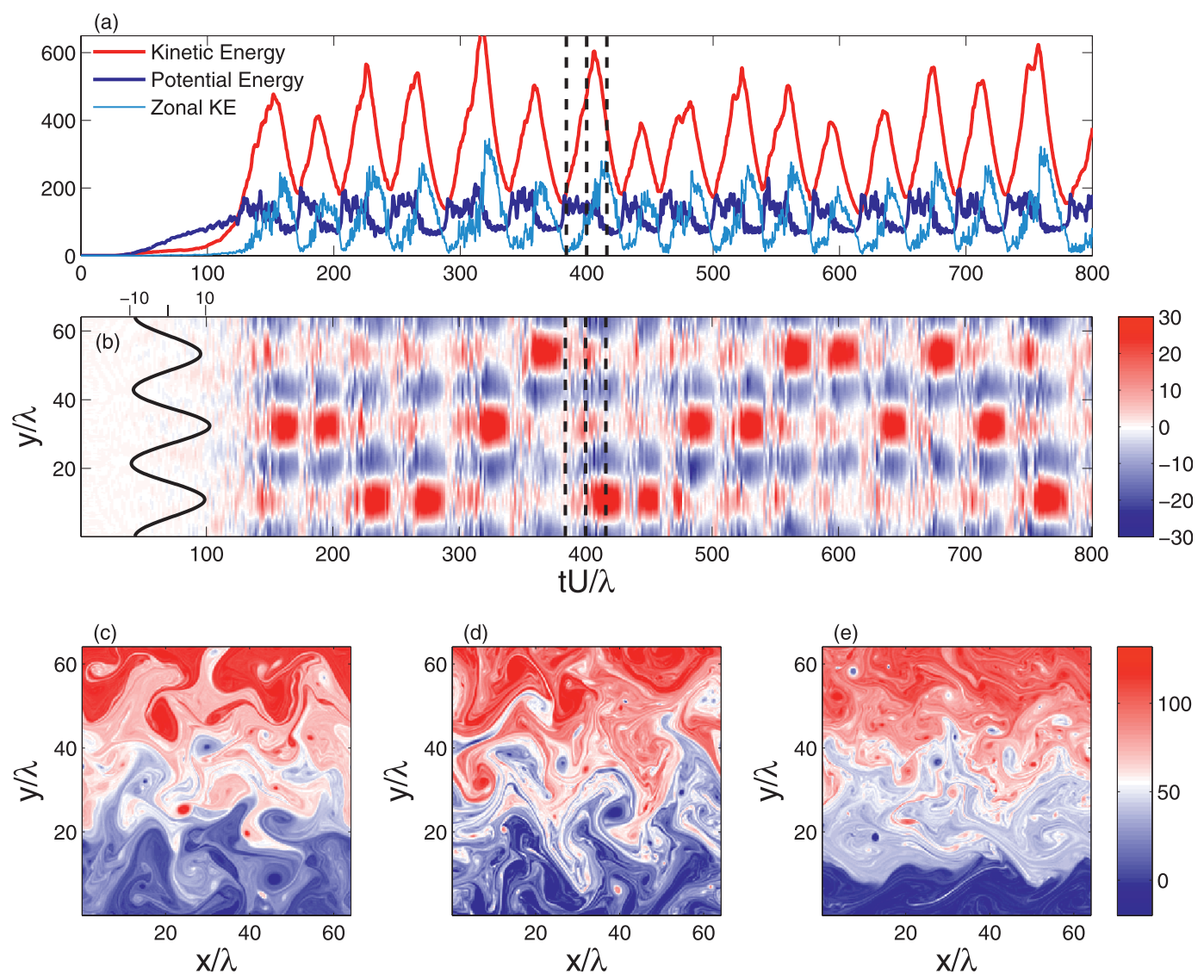

FIG. 12. (a) Time series of KE, PE, and ZKE for a simulation with $n_{B}=3, s=2.0$ (Fig. 8d). (b) Time series of zonally averaged barotropic zonal velocity, $u_{J}=-\bar{\psi}_{y}$, in the same simulation. (c)-(e) Snapshots of upper-layer PV at the times indicated by the dashed lines in (a) and (b).

a wider region. Figure 13a shows time series of $\ell_{\beta}$ from the simulation in Fig. 5b, estimated by dividing the domain into strong PV gradient regions $\left(\beta_{\mathrm{BT}}>11 / 12\right)$ and weak PV gradient regions $\left(\beta_{\mathrm{BT}}<7 / 12\right)$, where $\beta_{\mathrm{BT}}=$ $\beta+0.5(\partial h / \partial y)$. Different estimates of $\ell_{\beta}$ are calculated from the mean values of $\beta_{\mathrm{BT}}$ and $V_{e}$ in these separate regions. The eddy velocity scales $V_{e}$ are comparable in both strong and weak PV gradient regions, although $V_{e}$ experiences greater variability in regions of weak PV gradient (Fig. 13b). The relative contribution of turbulent motion and wave motion to the velocity field is also tied to the eddy scale. For example, where the PV gradient is stronger and $\ell_{\beta}$ is smaller, eddy energy is weaker and Rossby waves are more ordered, leading to stronger Reynolds stress correlations (Fig. 7). Thus, local PV modifications induced by topography are the primary means of generating spatially variable $\ell_{\beta}$, which leads to unsteady jet patterns.

The variability mechanism in the case with bumpy topography can be very different as evidenced by the energy diagnostics in Fig. 12, which undergo quasiperi- odic variability. In Fig. 14 a large number of snapshots have been collected from the experiment shown in Figs. $8 \mathrm{~d}$ and 12 over a period of $120 \lambda / U$. The mean streamfunction is calculated from a running mean of 20 snapshots spanning a period $5 \lambda / U$. This allows for an accurate calculation of the EKE and thus $\ell_{\beta}$. At the outset of a cycle (characterized by low-KE and high-PE values), $\ell_{\beta}$ is at a minimum and crucially, in these simulations, $\ell_{\beta} \leqslant \ell_{T}$. Thus, the jets feel the local PV modifications and are steered around the topographical features. During this stage, strong barriers to transport remain, despite the meandering. Nonzonal mean flows can generate eddy fields up to 100 times more energetic than eddy fields arising from purely zonal mean flows (Arbic and Flierl 2004). As described by Smith (2007), this strong generation arises because of correlations between zonal eddy velocities and eddy PV caused by jet-induced shear dispersion of eddies. Thus, topographic steering initiates a burst in EKE, which allows eddy length scales to grow through an inverse cascade until $\ell_{\beta}$ exceeds $\ell_{T}$. At this point, the controlling PV gradient is large-scale 

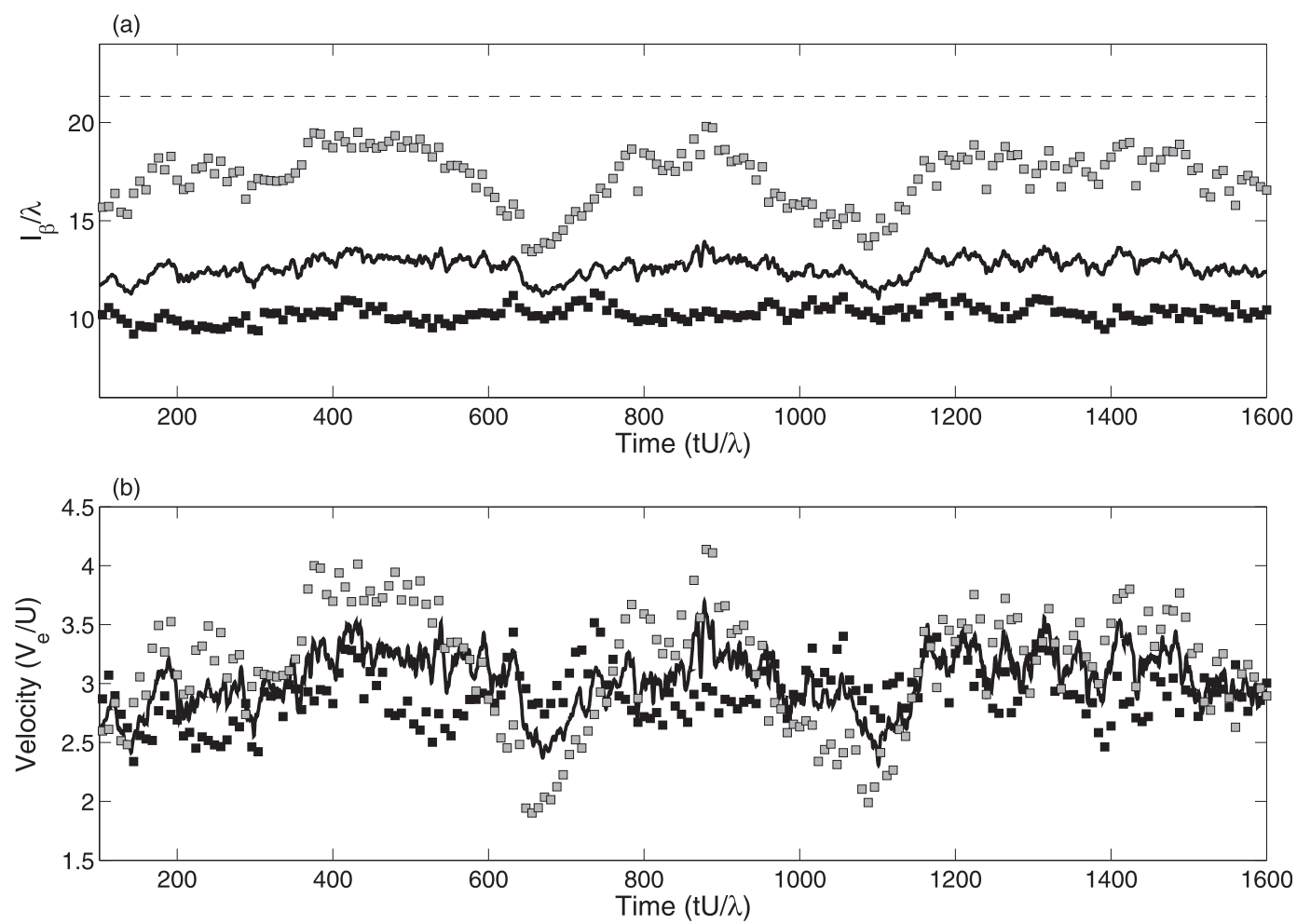

FIG. 13. (a) Estimates of $\ell_{\beta} / \lambda$ in a simulation with $n_{R}=3, s=1.0$ (Fig. 5b). The solid curve indicates $\ell_{\beta}$ based on domain-averaged values: $\ell_{\beta}=2 \pi \sqrt{V_{e} / \beta}$. The squares are values for $\ell_{\beta}^{\text {weak }}$ (gray) and $\ell_{\beta}^{\text {strong }}$ (black), where $V_{e}$ and $\beta_{\mathrm{BT}}$ are calculated by averaging over regions with weak $\left(\beta_{\mathrm{BT}}<7 / 12\right)$ and strong $\left(\beta_{\mathrm{BT}}>11 / 12\right) \mathrm{PV}$ gradients; $\beta_{\mathrm{BT}}=\beta+$ $0.5(\partial h / \partial y)$ takes values between $1 / 4$ and $5 / 4$ over the domain. The dashed line gives the value of $\ell_{T} / \lambda$. (b) The domainaveraged $V_{e}$ (solid line) as well as $V_{e}^{\text {weak }}$ (gray $\square$ s) and $V_{e}^{\text {strong }}$ (black $\square$ s) used in the top panel.

planetary $\beta$. Reynolds stress forcing of the mean flow acts preferentially in the zonal direction, and the flow becomes strongly zonal with $\ell_{\beta}>\ell_{T}$. As PE supplies are exhausted, $V_{e}$ decays and at some point $\ell_{\beta} \approx \ell_{T}$. Topographic steering resumes, which builds up PE stores and initiates the cycle again. Figure 14a also shows a time series of $\varkappa_{\mathrm{eff}}$, which indicates that the rapid growth of EKE is associated with bursts in turbulent mixing. This suggests that, in this regime at least, time- and domainaveraged meridional transports may be dominated by intermittent events, which are difficult to capture observationally or to parameterize in numerical models.

\section{b. Transport of passive tracers}

Effective diffusivity diagnostics calculated from the upper-layer PV give an accurate description of how a passive tracer, maintained by a large-scale gradient, would behave in this layer. There are subtle differences between passive tracers and PV in the lower layer because of bottom-friction effects. In particular, in flatbottom simulations, $\kappa_{\text {eff }}$ calculated in the lower layer from a passive tracer indicates mixing across eastward jets (similar to Greenslade and Haynes 2008), while a calculation using $Q_{2}$ indicates a barrier at the eastward jet, where bottom-friction effects are strongest. Still, the key point is that transport barriers diagnosed in the upper layer are a good representation of barriers associated with nearly barotropic jets (cf. Figs. 5 and 8).

The simulations documented here complicate the standard view of eastward jets as strict barriers to transport. Two paradigms are summarized by the schematic in Fig. 15. In the case where jets are zonally oriented, steady, domain-wrapping features (Fig. 15a), PV or tracers tend to become homogenized between the jets, with limited transport across the jets. If instead jets are continuously forming and merging, in either a temporal or streamwise sense (Fig. 15b), it becomes difficult to describe the meridional extent of a homogenized or well-mixed region. Furthermore, meridional jet migration offers a potential path for tracers to be carried through a latitude range greater than $\ell_{\beta}$ without ever crossing a sharp PV gradient. Thus, while jets may be effective transport barriers, over large times tracers may exhibit a greater meridional spread than would be implied by local eddy diffusivities. The effective diffusivity is a useful diagnostic, but it only provides a zonally averaged measure of mixing. Open 
(a)

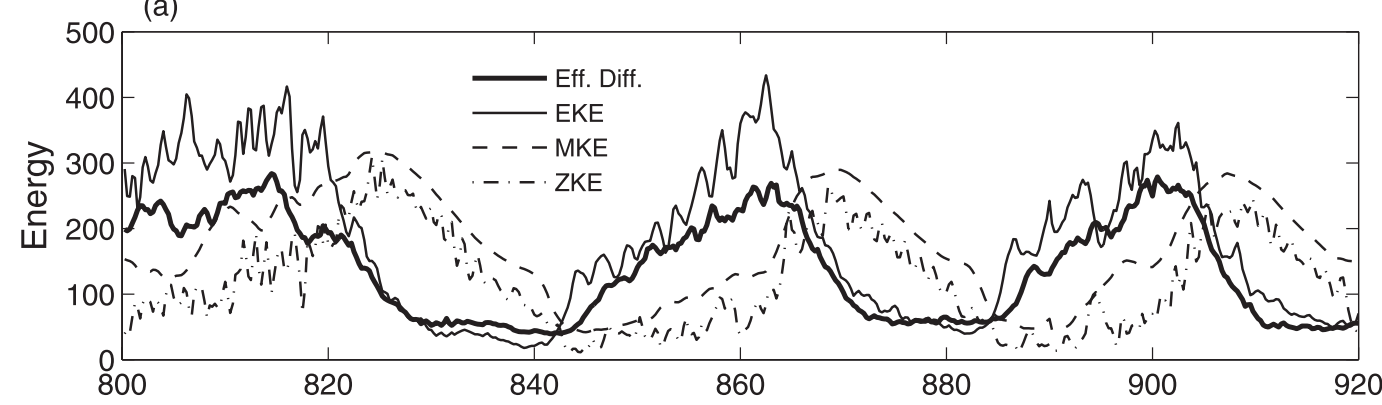

(b)

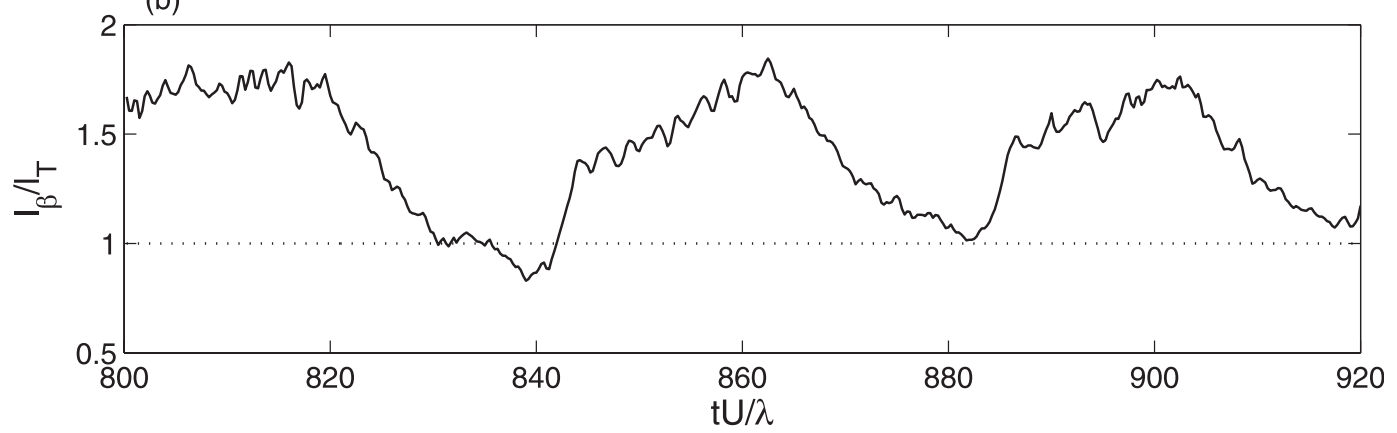

FIG. 14. (a) Time series of domain-averaged effective diffusivity $\varkappa_{\text {eff }}(16)$, mean kinetic energy (MKE), EKE, and ZKE for a simulation with $n_{B}=3, s=2.0$ (Fig. 8d). The mean fields are calculated from a 20-snapshot running mean spanning a period of $5 \lambda / U$. (b) Time series of the ratio $\ell_{\beta} / \ell_{T}$ from the same simulation.

questions remain as to whether topography generates preferred locations for meridional transport and, if so, how this relates to domain- or circumpolar-averaged measures of transport.

\section{c. Domain and resolution sensitivity}

Unsteady jet behavior may also occur in $\beta$-plane, baroclinic QG turbulence in a doubly periodic domain without topography because of quantization problems, since the system must accommodate an integer number of jets (Panetta 1993; Thompson and Young 2007). In this scenario, typically only an isolated region of the domain exhibits unsteady behavior. For example, if the system is varying between $n$ and $n+1$ jets, the system typically has $n-1$ steady jets and another wider jet that develops continuous branches. In certain cases, this branching can lead to meridional movement of the jets, but more often the variability persists along a constantlatitude band. Thus, there are some clear distinctions between the variability observed in flat-bottom simulations and topographically induced variability. However, to ensure that the variability described in this study is not attributable to domain constraints, especially when $\ell_{\beta}$ is comparable to $L$, sensitivity experiments were (a)

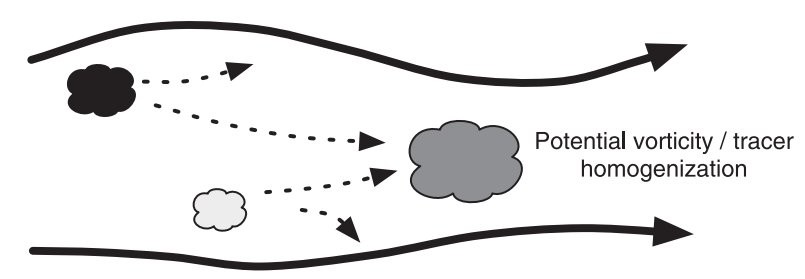

(b)

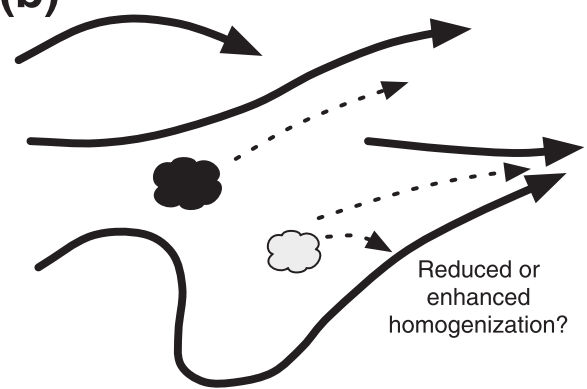

FIG. 15. (a) Schematic showing the traditional zonal jet paradigm that strong eastward flows act as barriers to transport, while the region between the jets is well mixed and becomes homogenized [see discussion of PV staircases in Dritschel and McIntyre (2008) and references therein]. (b) Schematic of the meandering and variable jet structures associated with the core of the ACC. The role of transport barriers in meridional mixing and property distributions remains poorly understood. 
TABLE 1. Statistics of the domain and resolution sensitivity studies. All runs have $\beta=0.75, \kappa=0.1$. KE is the total kinetic energy of the simulation, $\left\langle\psi_{x} \tau\right\rangle$ is the heat flux (8) \& (9), $\left\langle\bar{u}_{\max }\right\rangle$ is a time average of the maximum values of the zonally averaged zonal barotropic velocity $u$, and $\sqrt{\left\langle\bar{u}^{2}\right\rangle}$ is the rms value of this same velocity. The final column gives an estimate of the period of variability associated with either jet merger or jet oscillations.

\begin{tabular}{|c|c|c|c|c|c|c|c|}
\hline Expt No. & Resolution & Domain size $(\lambda)$ & $\langle K E\rangle / U^{2}$ & $\left\langle\psi_{x} \tau\right\rangle / U^{2} \lambda$ & $\left\langle\bar{u}_{\max }\right\rangle / U$ & $\sqrt{\left\langle\bar{u}^{2}\right\rangle} / U$ & Approx period $(\lambda / U)$ \\
\hline 32 & $256^{2}$ & 64 & 8.26 & 0.208 & 4.93 & 2.06 & 340 \\
\hline 32 & $512^{2}$ & 128 & 7.89 & 0.193 & 5.06 & 1.98 & 370 \\
\hline 32 & $512^{2}$ & 64 & 8.99 & 0.197 & 5.21 & 2.09 & 310 \\
\hline 32 & $1024^{2}$ & 64 & 8.13 & 0.203 & 4.70 & 2.02 & 360 \\
\hline 87 & $256^{2}$ & 64 & 331.9 & 13.6 & 24.7 & 10.5 & 41 \\
\hline 87 & $512^{2}$ & 128 & 279.6 & 11.6 & 29.0 & 9.16 & 52 \\
\hline 87 & $512^{2}$ & 64 & 337.9 & 13.8 & 24.7 & 10.4 & 46 \\
\hline 87 & $1024^{2}$ & 64 & 324.0 & 13.3 & 24.5 & 10.3 & 42 \\
\hline
\end{tabular}

conducted in wider domains $\left(128 \lambda^{2}\right)$ keeping all other parameters fixed. Simulations were also run to test the sensitivity of the results to the resolution. The sensitivity studies focused on the two main experiments discussed in this study: one producing braided jets (experiment 32, Fig. 5b) and one producing oscillating jets (experiment 87, Fig. 8d). The statistical properties and the frequency of the jet variability were comparable in the sensitivity simulations and are detailed in Table 1. There is no evidence that the dynamics in these simulations are controlled either by domain size or by resolution.

\section{d. Applicability to the Southern Ocean}

The dynamics of the two-layer QG simulations represent a substantial simplification over processes occurring in the ocean. While the model captures the most relevant dynamics (e.g., topographic steering, baroclinic instability, and jet sharpening through Reynolds stresses), much detail has been sacrificed for numerical efficiency that has allowed a broad sweep of parameter space and the cataloguing of different patterns of behavior. In particular, in a two-layer model, topography almost necessarily influences the equilibrated dynamics of the system. Even a modest increase in vertical resolution to a three-layer model may provide new results since eddy generation through baroclinic instability can proceed independent of topographical influences in the upper two layers.

While it is beyond the scope of the present study to explore all of the parameter space using a model with a greater vertical resolution, an important check on the relevance of the current study is reproducing similar dynamics in a model with three layers. To this end, a small number of doubly periodic, three-layer QG simulations with equal layer depths, also forced with an imposed vertically sheared mean flow, were run in similar parameter regimes; two of these simulations are shown in Fig. 16. The modification from two to three layers, not surprisingly, leads to changes in the equilibrated en- ergy levels-the three-layer model tends to be more energetic - and the frequency of the jet variability - the largest change is that the frequency of the jet merger in the ridge configuration is lower. Still, the key characteristics found in the two-layer model (e.g., braided and oscillating jets) are also observed in the three-layer model. In particular, the ordering of the lag in energy peaks in the case with bumpy topography (potentialkinetic-zonal) is maintained in the three-layer model. While it is reassuring that topographically induced jet variability is reproduced, it will still be necessary to verify that these dynamics are active in high-resolution ocean general circulation models or, even perhaps, using some combination of observational data.

Other important deviations of this idealized model from the real ocean include the method of forcing and the representation of topography. In the QG model, the amplitude and structure of the mean flow may adjust with time; however, the domain-averaged vertical shear is fixed. The baroclinicity in the ACC, on the other hand, arises due to surface convergences and divergences in the Ekman transport due to latitudinal variations in wind speed (Rintoul et al. 2001). Thus, additional variability in the jets may be introduced as the amplitude and orientation of the vertically sheared mean flow adapt to changes in the winds. One argument for the validity of the QG model used here is the recent suggestion that the ACC is in an eddy-saturated regime, whereby changes to the forcing are modulated by the eddy field, in which the baroclinic zonal mean flow is relatively insensitive to changes in wind forcing over long times (Hogg et al. 2008; Hallberg and Gnanadesikan 2006). In other words, the ACC adjusts through baroclinic instability to maintain a nearly constant mean vertical shear. A major restriction of QG theory with topography is that topographical variations must remain small as compared to the layer depth. That constraint has been enforced here by assuming that contributions to the PV gradient due to topography are comparable to 

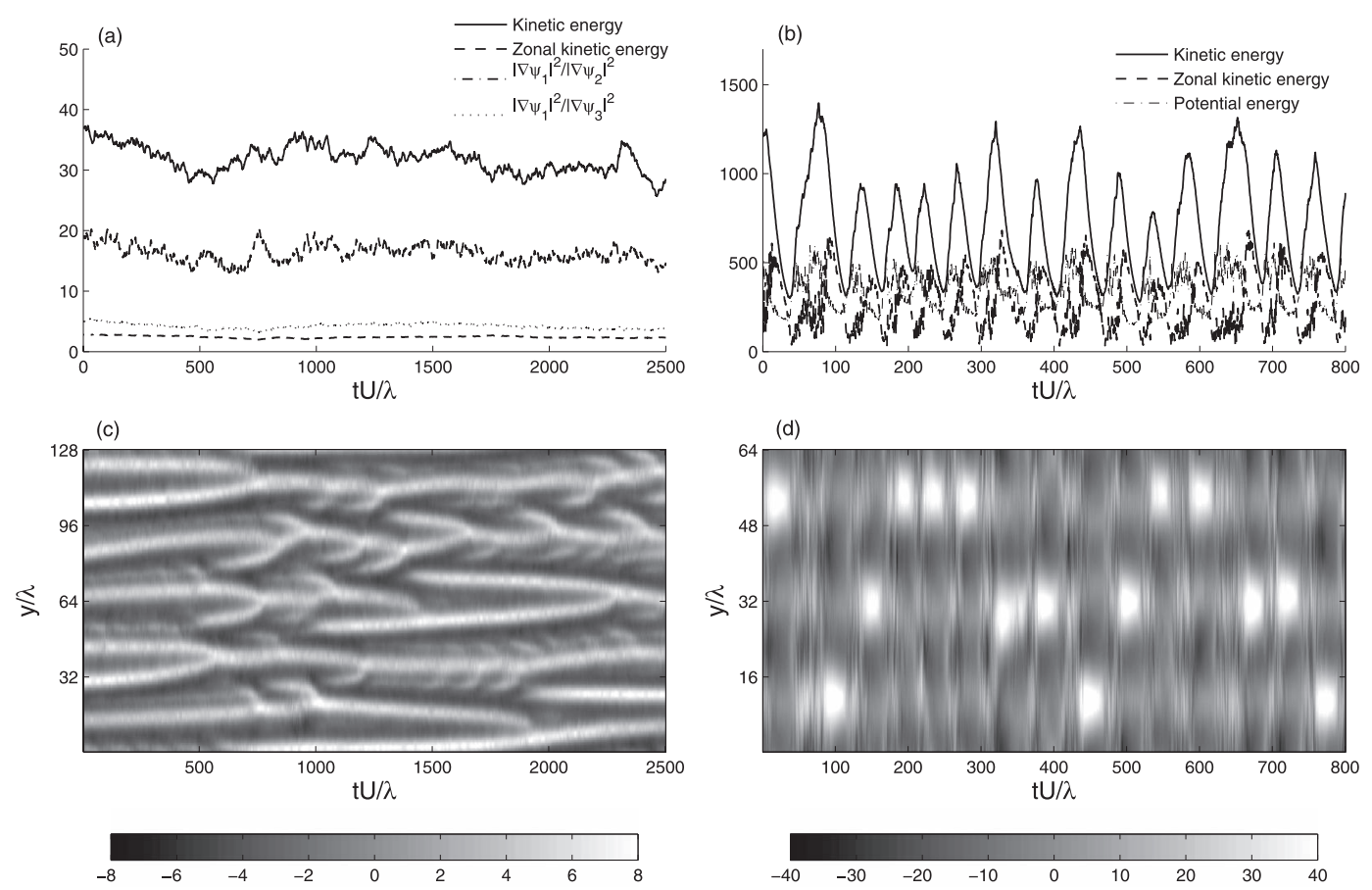

FIG. 16. (a) Time series of TKE (solid) and ZKE (dashed) in a three-layer simulation with zonal ridges (14) using $n_{R}=5, L=128 \lambda$, and $s=1.0$. The dashed-dotted and dotted lines show the ratios of upper-layer KE to middle- and lower-layer KE, respectively. (b) Time series of kinetic (solid), zonal (dashed), and potential (dashed-dotted) energies in a three-layer simulation with unaligned bumps (19) using $n_{B}=3, L=64 \lambda$, and $s=2.0$. (c) Time series of the zonally averaged zonal barotropic velocity for the simulation in (a). (d) Time series of the zonally averaged zonal barotropic velocity for the simulation in (b).

the contribution due to $\beta$. In reality, the ACC is dominated by steep topographical features, and the true QG limit may only apply in limited regions of the ACC. Still, the similarity between the simulations discussed here and altimetric observations (Hughes and Ash 2001; Sokolov and Rintoul 2007) hint that these dynamics may persist outside of the QG regime.

Finally, periodic behavior in QG simulations with topography has been observed in the work by Hogg and Blundell (2006), which focused on large-scale circulation patterns that experienced variability on decadal time scales. Using typical Southern Ocean values, $\lambda=$ $20 \mathrm{~km}$ and $U=20 \mathrm{~cm} \mathrm{~s}^{-1}$, the period $45 \lambda / U$ in Fig. 12 has a dimensional value of roughly 100 days. Thus, on much shorter time scales, or scales comparable to the variability observed in the narrow meandering jets of the ACC, interaction between $\ell_{\beta}$ and $\ell_{T}$ is a potential mechanism for local jet reorganization. Although the model used here is simplified, the evidence that these reorganization events are associated with bursts in meridional transport (cf. Fig. 8c), which may make a dominant contribution to the time-mean transports across the ACC, warrants further study into the role of smallscale jets on transport properties.

\section{Conclusions}

The spontaneous formation and evolution of jets on a doubly periodic $\beta$ plane with simple topography, forced by a baroclinically unstable vertical shear, has been explored. The sensitivity of meridional jet spacing, jet pattern variability, and domain-averaged meridional transport properties to variations in topographical length scale and steepness have been addressed. The main conclusions are as follow:

1) Jet spacing over a linear topographic slope $s$ in the lower layer is governed by the Rhines scale, $\ell_{\beta}=$ $2 \pi \sqrt{V_{e} / \beta_{\mathrm{BT}}}$, where $\beta_{\mathrm{BT}}=\beta+s / 2$ is the barotropic $\mathrm{PV}$ gradient.

2) Zonal ridges locally modify the meridional PV gradients and can reduce the domain-averaged meridional transport by up to a factor of $1 / 2$. Minimum energy levels occur when the Rhines scale is comparable to the topographic scale, $\ell_{\beta} \approx \ell_{T}$. When $\ell_{\beta}<$ $\ell_{T}$, the system experiences continuous jet formation and merger events contributing to a braided jet structure. The dependence of domain-averaged transport on $\ell_{T}$ is nonmonotonic in this regime. Regions where topography enhances the meridional 
PV gradient regulate the domain-averaged statistics of the flow.

3) Two-dimensional topography both modifies the local PV gradients and induces a nonzonal mean flow through topographic steering. In contrast to the ridge simulations, energy levels and meridional transport increase with increasing slope steepness, $s$. For fixed $s$, energy levels and meridional transport peak when $\ell_{\beta} \approx \ell_{T}$. Energy levels shift rapidly from a low-energy state to a high-energy state when $s$ is sufficiently large to induce local sign reversals in the meridional gradient of the barotropic PV. Transport barriers may exhibit steady, braided, and quasiperiodic behavior. In the latter case, the ratio $\ell_{\beta} / \ell_{T}$ oscillates between 1 and a value greater than 1 as more or less eddy kinetic energy is generated by baroclinic instability, based on the orientation of the mean flow. Meridional transport occurs in bursts in this case as jets reorganize frequently.

This study has provided a survey of mesoscale jet behavior over a range of simple topographies. Further work is required to fully understand the mixing and transport implications of some of the features described here, such as jet migration, jet merger, and meridional jet shifts. The ultimate goal is to understand how similar jet variability influences the meridional transport of heat, PV, and tracers in the ACC. An important result is that topography does not simply steer jets in a passive manner. Through modification of the mean flow and of local PV gradients and the subsequent feedback on baroclinic instability, topography can generate a range of patterns of unsteady jet behavior with significant consequences for large-scale transport properties. These processes are likely relevant to jet dynamics in the ACC.

Acknowledgments. The code used to calculate the effective diffusivities was provided by Peter Haynes. Insightful conversations with Pavel Berloff, Peter Haynes, Alberto Naveira Garabato, and David Stevens that improved this manuscript are gratefully acknowledged. This work was supported by a Natural Environment Research Council (NERC) Postdoctoral Fellowship.

\section{REFERENCES}

Allen, D. R., and N. Nakamura, 2001: A seasonal climatology of effective diffusivity in the stratosphere. J. Geophys. Res., 106, 7917-7935.

Arbic, B. K., and G. R. Flierl, 2004: Effects of mean flow direction on energy, isotropy, and coherence of baroclinically unstable betaplane geostrophic turbulence. J. Phys. Oceanogr., 34, 77-93.

Belkin, I. M., and A. L. Gordon, 1996: Southern Ocean fronts from the Greenwich meridian to Tasmania. J. Geophys. Res., 101, 3675-3696.
Berloff, P., I. Kamenkovich, and J. Pedlosky, 2009: A mechanism for formation of multiple zonal jets in the oceans. J. Fluid Mech., 628, 395-425, doi:10.1017/S0022112009006375.

Beron-Vera, F. J., M. G. Brown, M. J. Olascoaga, I. I. Rypina, H. Kocak, and I. A. Udovydchenkov, 2008: Zonal jets as transport barriers in planetary atmospheres. J. Atmos. Sci., 65, 3316-3326.

Borowski, D., R. Gerdes, and D. Olbers, 2002: Thermohaline and wind forcing of a circumpolar channel with blocked geostrophic contours. J. Phys. Oceanogr., 32, 2520-2540.

Charney, J. G., 1971: Geostrophic turbulence. J. Atmos. Sci., 28, 1087-1095.

Chelton, D. B., R. A. deSzoeke, M. G. Schlax, K. El Naggar, and N. Siwertz, 1998: Geographical variability of the first baroclinic Rossby radius of deformation. J. Phys. Oceanogr., 28, 433-460.

Dritschel, D. G., and M. E. McIntyre, 2008: Multiple jets as PV staircases: The Phillips effect and the resilience of eddytransport barriers. J. Atmos. Sci., 65, 855-874.

Dong, S., J. Sprintall, and S. T. Gille, 2006: Location of the Antarctic polar front from AMSR-E satellite sea surface temperature measurements. J. Phys. Oceanogr., 36, 2075-2089.

Gille, S. T., 2008: Decadal-scale temperature trends in the Southern Hemisphere ocean. J. Climate, 21, 4749-4765.

Greenslade, M. D., and P. H. Haynes, 2008: Vertical transition in transport and mixing in baroclinic flows. J. Atmos. Sci., 65, $1137-1157$.

Hallberg, R., and A. Gnanadesikan, 2006: The role of eddies in determining the structure and response of the wind-driven Southern Hemisphere overturning: Results from the Modeling Eddies in the Southern Ocean (MESO) project. J. Phys. Oceanogr., 36, 2232-2252.

Hart, J. E., 1975: Baroclinic instability over a slope. Part I: Linear theory. J. Phys. Oceanogr., 5, 625-633.

Haynes, P., and E. Shuckburgh, 2000: Effective diffusivity as a diagnostic of atmospheric transport 1. Stratosphere. J. Geophys. Res., 105, 22 777-22 794.

- D. A. Poet, and E. F. Shuckburgh, 2007: Transport and mixing in kinematic and dynamically consistent flows. J. Atmos. Sci., 64, 3640-3651.

Held, I. M., and D. G. Andrews, 1983: On the direction of eddy momentum flux in baroclinic instability. J. Atmos. Sci., 40, 2220-2231.

Hogg, A. McC., and J. R. Blundell, 2006: Interdecadal variability of the Southern Ocean. J. Phys. Oceanogr., 36, 1626-1645.

, M. P. Meredith, J. R. Blundell, and C. Wilson, 2008: Eddy heat flux in the Southern Ocean: Response to variable wind forcing. J. Climate, 21, 608-620.

Holloway, G., 1987: Systematic forcing of large-scale geophysical flows by eddy-topography interaction. J. Fluid Mech., 184, 463-476.

Holopainen, E. O., 1961: On the effect of friction in baroclinic waves. Tellus, 13, 363-367.

Huang, H.-P., and W. A. Robinson, 1998: Two-dimensional turbulence and persistent zonal jets in a global barotropic model. J. Atmos. Sci., 55, 611-632.

Hughes, C. W., and E. R. Ash, 2001: Eddy forcing of the mean flow in the Southern Ocean. J. Geophys. Res., 106, 2713-2722.

Kamenkovich, I., P. Berloff, and J. Pedlosky, 2009: Role of eddy forcing in the dynamics of multiple zonal jets in the North Atlantic. J. Phys. Oceanogr., 39, 1361-1379.

Larichev, V., and I. Held, 1995: Eddy amplitudes and fluxes in a homogeneous model of fully developed baroclinic instability. J. Phys. Oceanogr., 25, 2285-2297. 
Lee, M.-M., and A. C. Coward, 2003: Eddy mass transport for the Southern Ocean in an eddy-permitting global ocean model. Ocean Modell., 5, 249-266.

Le Quéré, C., and Coauthors, 2007: Saturation of the Southern Ocean $\mathrm{CO}_{2}$ sink due to recent climate change. Science, 316, $1735-1738$.

Marshall, J., E. Shuckburgh, H. Jones, and C. Hill, 2006: Estimates and implications of surface eddy diffusivity in the Southern Ocean derived from tracer transport. J. Phys. Oceanogr., 36, 1806-1821.

Maximenko, N. A., B. Bang, and H. Sasaki, 2005: Observational evidence of alternating zonal jets in the world oceans. Geophys. Res. Lett., 32, L12607, doi:10.1029/2005GL022728.

Munk, W. H., and E. Palmén, 1951: Note on the dynamics of the Antarctic Circumpolar Current. Tellus, 3, 53-55.

Nakamura, N., 1996: Two-dimensional mixing, edge formation, and permeability diagnosed in area coordinates. J. Atmos. Sci., 53, 1524-1537.

, 2008a: Quantifying inhomogeneous, instantaneous, irreversible transport using passive tracer field as a coordinate. Transport and Mixing in Geophysical Flows, J. B. Weiss and A. Provenzale, Eds., Lecture Notes in Physics, Vol. 744, Springer-Verlag, 137-164.

- 2008b: Sensitivity of global mixing and fluxes to isolated transport barriers. J. Atmos. Sci., 65, 3800-3818.

Nakano, H., and H. Hasumi, 2005: A series of zonal jets embedded in the broad zonal flows in the Pacific obtained in eddypermitting ocean general circulation models. J. Phys. Oceanogr., 35, 474-488.

Olbers, D., D. Borowski, C. Völker, and J.-O. Wölff, 2004: The dynamical balance, transport and circulation of the Antarctic Circumpolar Current. Antarct. Sci., 16, 439-470.

Orsi, A. H., T. W. Whitworth III, and W. D. Nowlin Jr., 1995: On the meridional extent and fronts of the Antarctic Circumpolar Current. Deep-Sea Res., 42, 641-673.

- S. S. Jacobs, A. L. Gordon, and M. Visbeck, 2001: Cooling and ventilating the abyssal ocean. Geophys. Res. Lett., 28, 2923-2926.

Panetta, R. L., 1993: Zonal jets in wide baroclinically unstable regions: Persistence and scale selection. J. Atmos. Sci., 50, 2073-2106.

Pavan, V., and I. M. Held, 1996: The diffusive approximation for eddy fluxes in baroclinically unstable jets. J. Atmos. Sci., 53, $1262-1272$.

Pedlosky, J., 1987: Geophysical Fluid Dynamics. 2nd ed. SpringerVerlag, $710 \mathrm{pp}$.

Rhines, P. B., 1994: Jets. Chaos, 4, 313-339.

Richards, K. J., N. A. Maximenko, F. O. Bryan, and H. Sasaki, 2006: Zonal jets in the Pacific Ocean. Geophys. Res. Lett., 33, L03605, doi:10.1029/2005GL024645.
Rintoul, S. R., C. W. Hughes, and D. Olbers, 2001: The Antarctic Circumpolar Current system. Ocean Circulation and Climate, G. Siedler, J. Church, and J. Gould, Eds., Academic Press, 271-302.

Shuckburgh, E., and P. Haynes, 2003: Diagnosing transport and mixing using a tracer-based coordinate system. Phys. Fluids, 15, 3342-3357.

Sinha, B., and K. J. Richards, 1999: Jet structure and scaling in Southern Ocean models. J. Phys. Oceanogr., 29, 1143-1155.

Smith, K. S., 2007: Eddy amplitudes in baroclinic turbulence driven by nonzonal mean flow: Shear dispersion of potential vorticity. J. Phys. Oceanogr., 37, 1037-1050.

— , and J. Marshall, 2009: Evidence for deep eddy mixing in the Southern Ocean. J. Phys. Oceanogr., 39, 50-69.

—- G. Boccaletti, C. C. Henning, I. Marinov, C. Y. Tam, I. M. Held, and G. K. Vallis, 2002: Turbulent diffusion in the geostrophic inverse cascade. J. Fluid Mech., 469, 13-48.

Sokolov, S., and S. R. Rintoul, 2007: Multiple jets of the Antarctic Circumpolar Current south of Australia. J. Phys. Oceanogr., 37, 1394-1412.

Spall, M. A., 2000: Generation of strong mesoscale eddies by weak ocean gyres. J. Mar. Res., 58, 97-116.

Steinsaltz, D., 1987: Instability of baroclinic waves with bottom slope. J. Phys. Oceanogr., 17, 2343-2350.

Thompson, A. F., and W. R. Young, 2006: Scaling baroclinic eddy fluxes: Vortices and energy balance. J. Phys. Oceanogr., 36, $720-738$.

— , and — 2007: Two-layer baroclinic eddy heat fluxes: Zonal flows and energy balance. J. Atmos. Sci., 64, 3214-3231.

Treguier, A. M., and B. L. Hua, 1988: Influence of bottom topography on stratified quasi-geostrophic turbulence. Geophys. Astrophys. Fluid Dyn., 43, 265-305.

_ , and J. C. McWilliams, 1990: Topographic influences on winddriven, stratified flow in a $\beta$-plane channel: An idealized model for the Antarctic Circumpolar Current. J. Phys. Oceanogr., 20, 321-343.

—, and R. L. Panetta, 1994: Multiple zonal jets in a quasigeostrophic model of the Antarctic Circumpolar Current. J. Phys. Oceanogr., 24, 2263-2277.

Vallis, G. K., and M. E. Maltrud, 1993: Generation of mean flows and jets on a beta plane and over topography. J. Phys. Oceanogr., 23, 1346-1362.

Williams, R. G., C. Wilson, and C. W. Hughes, 2007: Ocean and atmosphere storm tracks: The role of eddy vorticity forcing. J. Phys. Oceanogr., 37, 2267-2289.

Wolff, J.-O., E. Maier-Reimer, and D. J. Olbers, 1991: Wind-driven flow over topography in a zonal $\beta$-plane channel: A quasigeostrophic model of the Antarctic Circumpolar Current. J. Phys. Oceanogr., 21, 236-264. 